\title{
VCP protects neurons from proteopathic seeding
}

Jiang Zhu ${ }^{1}$, Sara Pittman ${ }^{1}$, Dhruva Dhavale ${ }^{1}$, Rachel French ${ }^{2}$, Jessica N. Patterson ${ }^{1}$, Salman

Kaleelurrrahuman ${ }^{1}$, Yuanzi Sun ${ }^{4}$, William J. Buscher ${ }^{3}$, Jan Bieschke ${ }^{4}$, Paul Kotzbauer ${ }^{1}$, Yuna Ayala $^{2}$, Albert A. Davis ${ }^{1}$, Conrad Weihl ${ }^{1 *}$

${ }^{1}$ Department of Neurology, Hope Center for Neurological Diseases, Washington University School of Medicine, St Louis, MO, USA

${ }^{2}$ Edward A. Doisy Department of Biochemistry and Molecular Biology, Saint Louis University School of Medicine, St. Louis, MO, USA.

${ }^{3}$ Department of Genetics, Washington University School of Medicine, St. Louis, MO, USA

${ }^{4}$ Medical Research Council Prion Unit / UCL Institute of Prion Diseases, University College London, London, UK;

Corresponding author and Lead contact:

${ }^{*}$ Conrad C. Weihl

Department of Neurology, Hope Center for Neurological Diseases, Washington University

School of Medicine, St Louis, MO 63110, USA

Tel: 314-362-6981; fax: 314-362-3752

E-mail: weihlc@wustl.edu 


\section{SUMMARY}

Uptake and spread of proteopathic seeds, such as aS, Tau, and TDP-43, contribute to neurodegeneration. The cellular machinery necessary for this process is poorly understood. Using a genome-wide CRISPR-Cas9 screen, we identified Valosin Containing Protein (VCP) as a suppressor of aS seeding. Dominant mutations in VCP cause multisystem proteinopathy (MSP) with muscle and neuronal degeneration. VCP inhibition or disease mutations increase aS seeding in cells and neurons. This is not associated with an increase in seed uptake and is similar to treatment with the lysosomal damaging agent, LLoME. Intrastriatal injection of aS seeds into VCP disease mice enhances seeding efficiency compared with controls. This is not specific to aS since VCP inhibition or disease mutations increased TDP-43 seeding in neurons. These data support that VCP protects against proteopathic spread of pathogenic aggregates. The spread of distinct aggregate species may dictate pleiotropic phenotypes and pathologies in VCP associated MSP. 


\section{INTRODUCTION}

a-synuclein (aS) is the principal component of protein inclusions that are found in a family of neurodegenerative disorders known as synucleinopathies ${ }^{1}$. Synucleinopathies include Parkinson's Disease, Diffuse Lewy body disease (DLB), multiple system atrophy and REM sleep behavior disorder (RBD) ${ }^{1}$. aS contains an amyloid-like region making it prone to aggregate. Several lines of evidence suggest that aggregated aS can seed the fibrillization of soluble, monomeric $a S^{2}$. This process may relate to disease pathogenesis and progression ${ }^{3}$. aS fibrils enter neurons via endocytosis and template new aS aggregates within the cytoplasm 4. This leads to synapse loss, neurodegeneration, and ultimately the release of aS fibrils to adjacent cells resulting in aggregate propagation along interconnected neurons ${ }^{3}$. The cellular process of proteopathic seeding consists of several regulated steps. These include seed uptake, vesicular trafficking, endolysosomal escape and templated conversion of cytosolic aS ${ }^{5}$.

One route of seed uptake is endocytosis. aS seeds can enter cells and neurons via the endocytic system. These seeds then penetrate the endolysosomal membrane facilitating their escape into the cytoplasm ${ }^{5}$. Pharmacologically blocking cellular uptake or increasing endolysosomal membrane damage can decrease and increase seeding efficiency respectively

5. Previous studies have identified genetic modifiers of aS toxicity associated with its intracellular expression in yeast or $C$. elegans ${ }^{6}$. However, genetic modifiers of proteopathic seeding have not been explored.

VCP (also called p97, or cdc48 in yeast) is a ubiquitin-directed AAA-ATPase implicated in multiple forms of neurodegeneration ${ }^{7}$. Dominantly inherited mutations in VCP cause multisystem proteinopathy (MSP) which is associated with multiple variably penetrant phenotypes that include inclusion body myopathy, frontotemporal dementia, ALS, and Parkinsonism ${ }^{7}$. Just 
as the phenotypes are variable, VCP patients develop varied aggregate pathologies that include aS, TDP-43, Tau, SQSTM1 and ubiquitin inclusions ${ }^{8-11}$. How VCP disease mutations lead to cellular degeneration and protein inclusions is unclear. VCP affects the trafficking and clearance of polyQ aggregates in vitro ${ }^{12}$. VCP is also necessary for both autophagic and proteasomal degradation of ubiqutinated proteins including TDP-43 and ER associated proteins via ERAD ${ }^{7}$. VCP has more recently been proposed to behave as a protein disaggregase specifically acting upon pathologic tau aggregates ${ }^{11}$. By binding to distinct adaptors, VCP alters it functionality allowing it to participant in its many other functions such as ERAD, vesicular trafficking, DNA repair, and cell cycle regulation ${ }^{7}$.

VCP disease mutations alter its association with distinct adaptors ${ }^{13,14}$. Specifically, VCP disease mutations have reduced binding to UBXD1 and increased interactions with Ufd1/Npl4 creating both a loss and gain of function with regard to UBXD1 and Ufd1 dependent processes 13-16. Notably, a VCP-UBXD1 dependent complex is recruited to damaged endolysosomes ${ }^{15}$. This complex recruits the deubiquitinase YOD1 which cleaves K48 linked ubiquitin chains from the lysosomal membrane facilitating lysophagic degradation ${ }^{15}$. VCP inhibition, loss of UBXD1, or VCP disease mutant expression lead to a delay in the clearance of damaged late endosomes resulting in the accumulation of galectin-3 positive puncta in both VCP mouse models and patient tissue ${ }^{15,17}$.

MSP patients are pathological characterized as a TDP-43 proteinopathy ${ }^{18}$. MSP patient tissue accumulates aggregated and insoluble TDP-43 in affected muscle and CNS tissue ${ }^{18} .90 \%$ of MSP patients have myopathy that precedes dementia by $\sim 10$ years ${ }^{19}$. Whether TDP-43 aggregate pathology spreads from muscle to motor neuron and ultimately the cortex is not known. TDP-43 contains an intrinsically disordered or prion-like domain that facilitates its 
templated aggregate conversion ${ }^{20}$. Like aS, TDP-43 aggregates can serve as proteopathic seeds that propagate in cell and mouse models ${ }^{21,22}$.

Functional genomic screens are a powerful tool to identify proteins participating in distinct cellular pathways. In this study, we utilized an aS seeding FRET biosensor to screen a CRISPR knockout library. This approach identified multiple suppressors of aS seeding of which the AAA ATPase, VCP, was further explored both in vitro and in vivo. 


\section{RESULTS}

Genome-wide CRISPR knockout screen identifies genes protective against aS seeding.

To identify genes that regulate aS seeding, we performed a genome-wide screen in a previously described HEK293 aS CFP/YFP biosensor cell line (aS biosensor) (Figure 1A) ${ }^{4}$. Exogenously applied pre-formed fibrillar aS (aS PFF), but not monomeric aS, seeds the aggregation of soluble intracellular aS resulting in a quantifiable change in FRET efficiency. We clonally expressed spCAS9 in aS biosensors and then infected with a pooled Brunello gRNA library covering 19114 different genes/ 4 gRNA each and 1000 non-targeting controls at a low MOI $(<0.3)$ for seven days with puromycin selection. The pooled knockdown aS biosensor maintained its normal aS seeding capacity (Figure S1). These biosensors were treated with aS PFF as previously described and flow-sorted into FRET positive and FRET negative groups. DNA was isolated from FRET positive, FRET negative, and the unseeded total cell population. Then next-generation sequencing was performed to identify the guide RNAs (gene knockdowns) represented in each group. This sequence data was analyzed via the MegaCK algorithm in both the FRET positive and negative populations compared with untreated control. 110 genes were enriched in the FRET positive population as compared to total population, and 43 genes were underrepresented in the FRET negative group versus total population (FDR $<0.05$ and fold change $>2$ or $<0.5$ ) (Figure 1B). These 153 genes were considered "protective" or suppressors of aS seeding in the biosensor line (Figure 1B-C; Table S1).

Our screen identified genes and pathways previously identified in other screens for aS toxicity. Notably, we identified 15 genes associated with ER-Golgi-endosome trafficking. These included VPS51 and VPS52 which are components of the Golgi-associated retrograde protein (GARP) complex. The GARP complex interacts with PD associated protein, LRRK2, and deletion of either VPS51 or VPS52 homologs in yeast, increases aS accumulation and toxicity ${ }^{23}$. Other 
modifiers not previously identified in screens include ATP6V0B, ATP6V0C, and ATP6V1A that encode subunits of Vacuolar-type ATPase (V-ATPase). ATP6V0B KD inhibits autophagic degradation and increases aS aggregation ${ }^{24}$ and is downregulated in patients with aS inclusions ${ }^{25}$. Pathway analysis identified an enrichment in genes associated with the cellular stress response such as VCP, SEC61B and KDELR1. Notably the ER stress response is upregulated in PD brains and correlates with aS toxicity in multiple model systems ${ }^{26}$.

We validated nine candidate suppressors (ATP6V0C, KDELR1, LAMTOR5, RAB35, RABAC1, SEC61B, TMEM147, VCP and VPS51) using siRNA knockdown in aS biosensors. Following 48 hours of siRNA treatment, aS PFF was added with Lipofectamine, and FRET efficiency was measured 24 hours later. Six candidate suppressors, when knocked down, increased FRET efficiency and included ATP6V0C, VPS51, KDELR1, SEC61B, LAMTOR5 and VCP (Figure 1D). To further confirm our findings with VCP, we generated a lentiviral vector expressing a VCP specific gRNA or control, infected spCAS9 aS biosensors for 7 days and treated with aS PFF. Similar to that seen with VCP siRNA, FRET efficiency was increased in VCP CRISPR KO aS biosensors (Figure 1E-F).

VCP inhibition increases a-synuclein seeding efficiency.

As previously reported, aS seeding as measured by FRET is not seen with the application of monomeric aS ${ }^{4}$. Moreover, Lipofectamine is necessary for efficient FRET. The application of "naked" aS PFF to aS biosensors fails to increase FRET after 24 and 48 hours suggesting that Lipofectamine facilitates entry into the endolysosomal pathway ${ }^{4}$. Consistent with this, the enhanced seeding efficiency and FRET signal following VCP siRNA treatment required the application of aS PFF with Lipofectamine and did not occur with monomeric aS or when aS PFF were added without Lipofectamine (Figure 2A). 
In order to probe the role of VCP specifically at the time of endocytic entry into the cytosol, we modified our seeding protocol to a four-hour application with lipofectamine and subsequent washout of aS PFF from the media and determined this to be sufficient to seed aS aggregation as measured by FRET in a concentration-dependent manner at 24 hours (Figure S2A). Consistent with aS PFF entry through the endolysosomal pathway, treatment of aS biosensors with Dynogo-4a, a dynamin I/II inhibitor with aS PFF decreased FRET. Whereas a four-hour treatment at the time of aS PFF application with the lysosomal permeabilizing agent (LLoMe) significantly increased FRET (Figure 2B). Treatment of aS biosensors with the VCP inhibitor NMS-873 for four hours at the time of aS PFF application similarly increased seeding efficiency as measured by FRET (Figure 2B). The application of two additional VCP inhibitors, CB-5083, and Eeyarestatin I (Eer1) during aS PFF seeding also increased seeding efficiency measured by FRET (Figure 2B). This effect was aS PFF dependent since the application of VCP inhibitors or LLoMe in the presence of aS monomer did not increase FRET (Figure 2B). A similar experiment adding the polyphenol (-)-epigallocatechin gallate (EGCG), an anti-amyloid agent at the time of aS PFF application, resulted in a decrease in seeding efficiency (Figure 2B). Finally, the application of the proteasome inhibitor bortezomib or autophagy modulators (Rapamycin and 3-methyladenine (3-MA)) did not affect seeding efficiency (Figure 2B).

To see whether the increased seeding efficiency with VCP inhibition was due to an increase in aS PFF uptake, we employed a fluorescently conjugated aS PFF (aS-PFF 647). aS-PFF 647 retains seeding capacity in aS biosensors (Figure S2B). A four-hour aS biosensor treatment with aS-PFF 647 in the presence of LLoME or NMS-873 did not increase the amount of internalized aS PFF 647 (Figure 2C-D). Uptake was also unchanged when comparing scrambled and VCP siRNA KD (Figure 2E). In contrast, the application of Dynogo-4a 
significantly decreased aS PFF 647 uptake (Figure 2F). In addition, the increased seeding efficiency with VCP chemical inhibition or VCP siRNA knockdown was not due to an increase in the steady-state levels of aS as determined by immunoblot and aS fluorescence intensity via flow cytometry (Fig 2G-K). VCP inhibition and knockdown induce ER stress and activate the unfolded protein response (UPR) ${ }^{27,28}$. Treatment of aS biosensors with the ER stress inducing agents dithiothreitol (DTT), thapsigargin and tunicamycin for 24 hours following the application of aS PFF decreased FRET efficiency as compared to vehicle controls suggesting that the effect of VCP inhibition on seeding was ER stress independent (Figure 2L).

To explore the role of VCP in a more relevant system of aS seeding that does not require the use of the carrier Lipofectamine, we adapted a previously described assay which adds aS PFF to primary cultured hippocampal neurons $(\mathrm{HNs})^{29}$. The addition of aS PFF to the media of HNs for four hours, followed by media exchange, resulted in detergent-insoluble, high molecular weight $\alpha S$, and phospho- $a S$ as measured by fractionation immunoblot or via immunofluorescence using a phospho-aS antibody in HNs after five days (Figure 3A-B). The coapplication of LLoMe and aS PFF for 4 hours followed by washout further increased the amount of phospho-aS as compared with the application of aS PFF and vehicle after five days (Figure 3B-C). These results were aS PFF dependent since monomeric aS failed to generate phosphoaS staining (Figure S3A). We performed a similar assay and treated HNs for four hours with the reversible VCP inhibitor ML240 and aS PFF. Notably, the application of ML240 for four hours at the time of aS PFF application increased the level of phospho-aS as compared with vehicletreated control, while drug treated HNs with aS monomers show no phospho-aS (Figure 3D-E, S3A). Treatment of primary HNs with two different shRNAs against VCP for four days before seed application further demonstrated an increase in an aS PFF-dependent increase in phospho-aS staining compared with scrambled shRNA control (Figure 3F-G; S3B). 


\section{VCP disease mutation expression increases aS seeding.}

VCP disease mutations affect a subset of VCP dependent cellular processes such as endocytic trafficking, nutrient sensing, autophagosome maturation, and, more recently, lysophagy ${ }^{7,15}$. This is due to an impairment in VCP mutant association with the adaptor UBXD1. We performed siRNA knockdown of VCP and the VCP adaptors UFD1, NPL4, UBXD1, and PLAA and the autophagy proteins ATG5 and SQSTM1 along with a scrambled control in aS biosensors. Following 48 hours of knockdown, aS biosensors were treated with aS PFF, and FRET was measured 24 hours later. Knockdown of VCP or its adaptor UBXD1 significantly increased FRET efficiency above controls (Figure 4A and S4A). shRNA knockdown of UBXD1 in primary HNs 4 days prior to aS PFF application also increased phospho-aS staining as compared with scrambled shRNA control (Figure 4B-C). To understand if VCP disease mutant expression increased aS seeding, similar to VCP and UBXD1 knockdown, we transfected aS biosensors with mCherry-tagged VCP-WT or one of three different VCP disease mutations (R95G, R155H, and A232E) for 24 hours. Treatment with aS PFF and quantitation of FRET efficiency 24 hours later in mCherry positive cells demonstrated an increase in FRET in VCP disease mutant expressing cells compared with VCP-WT control. Whereas cells not expressing mCherry did not show differences (Figure 4D and S4C).

VCP-R155H mutation knockin mice have been previously generated and characterized ${ }^{30}$. We cultured primary HNs from VCP ${ }^{\mathrm{WT} / \mathrm{WT}}$ and $\mathrm{VCP} \mathrm{PH}^{\mathrm{RHT}}$ embryos, treated them with aS PFF and then immunostained for phospho-aS five days later. VCP ${ }^{\mathrm{RH} W \mathrm{NT}}$ had a significant increase in phospho-aS staining compared with VCP ${ }^{\mathrm{WT} / \mathrm{WT}}$ controls (Figure 4E-F). 
Using the same VCP-R155H mutation knockin mice, we examined the effect of pathogenic VCP mutations on aS seeding in vivo. VCP ${ }^{\mathrm{RH} / \mathrm{WT}}$ mice display no neuronal loss, TDP-43 inclusions, or pathologic features consistent with autophago-lysosomal dysfunction up to 13 months old (paper in print). To explore an additional VCP mouse model that only expresses a VCP disease mutant allele, we also used a mouse line that deletes the VCP-WT allele and only allows expression of a single VCP-R155C mutant allele following tamoxifen treatment $\left(\mathrm{VCP} \mathrm{RC}^{\mathrm{FL}}\right.$; Rosa26-Cre ERT2 (cVCP-RC) (Figure S5) (paper in print). Similar to our previous study, lysates from the cortex of VCP ${ }^{\mathrm{RH} / \mathrm{WT}}$ have no changes in the levels of autophagic (LC3, Sequestosome-1/p62), ER stress (BiP/GRP78), or ubiquitinated proteins (Figure 5A-B). Following five days i.p. tamoxifen treatment, cortical lysates from cVCP-RC mice have a $41 \%$ reduction in total VCP protein level but no changes in autophagic levels (LC3, Sequestosome1/p62), ER stress (BIP/GRP78) or ubiquitinated proteins (Figure 5A-B). However, high molecular weight ubiquitinated proteins and SQSTM1 levels increase with age as demonstrated by immunoblot of cortical lysates at 6 months supporting that VCP dysfunction is present (Figure 5C-D). We have previously demonstrated that an increase in Gal3 levels occurs early before autophagic dysfunction in VCP ${ }^{\mathrm{RH} / \mathrm{WT}}$ mouse muscle ${ }^{17}$. Similar to skeletal muscle, Gal3 and LAMP1 levels are increased in both VCP ${ }^{\mathrm{RH} W T}$ and cVCP-RC mouse cortical lysates suggesting an accumulation of damaged late endosomes (Figure $5 A-B){ }^{15,17}$.

We injected 5ug aS PFF or PBS into the striatum of 4-month-old C57 control, VCP ${ }^{\mathrm{RH} / \mathrm{WT}}$, or cVCP-RC mice and harvested the brain after 3 months (Figure 6A). Untreated control, $V_{C P}^{R H / W T}, c V C P-R C$ mice, or mice treated with PBS had no phospho-aS staining in any brain regions (Figure 6A-E). In contrast, C57 control mice injected with aS PFF had a significant increase of phospho-aS in multiple brain regions (Figure 6A-E). This increase was significantly increased above that of injected C57 mice in the anterior and posterior cortices of VCP ${ }^{\mathrm{RH} / \mathrm{WT}}$, and 
cVCP-RC injected with aS PFF (Figure 6A-E). Other brain regions such as the amygdala and substantia nigra trended toward an increase in phospho-aS staining but did not reach statistical significance (Figure 6F-G).

\section{VCP disease mutations enhance TDP-43 seeding.}

A subset of VCP patients have Parkinsonism and post-mortem evidence of aS pathology, however, most patients have TDP-43 inclusions in the CNS and muscle ${ }^{8-10}$. To evaluate the role of VCP in the seeding of TDP-43, we developed a TDP-43 seeding assay in primary hippocampal neurons. The addition of TDP PFF to HNs resulted in the appearance of phosphorylated TDP-43 Ser409/410 (pTDP) positive puncta in a concentration-dependent manner (Figure 7A-B) and redistribution of pTDP from the nucleus to the cytoplasm (Figure 7C) after 5 days. PTDP staining was not increased or altered when HNs were treated with aS PFFs (Figure S6). Fractionation of lysates from HNs one or 5 day post-treatment with buffer, nonaggregated TDP-43 (monomeric TDP-43), or TDP-43 aggregated at room temperature for 24 hours (TDP-43 PFF) and subsequent immunoblot for pTDP revealed an increase in high molecular weight TDP-43 in the RIPA insoluble fraction of TDP PFF treated HNs (Figure 7D). In addition, TDP-43 PFF induced cytosolic pTDP-43 puncta that co-localized with Sequestosome-1 and TIA1 similar to pathologic TDP-43 inclusions in patients (Figure 7E). As seen with VCP mutant expression in TDP-43 biosensors, treatment of primary HNs from VCPWTNT and VCP ${ }^{R H / W T}$ embryos with TDP-43 PFF revealed an increase pTDP-43 puncta in VCP ${ }^{R H / W T}$ HNs compared with VCP ${ }^{\text {WT/NT }}$ HNs (Figure 7F-G). 


\section{DISCUSSION}

Functional genomic screens utilizing CRISPR knockout approaches are an invaluable resource to elucidate proteins related to distinct cellular pathways. Here, we employed a CRISPR wholegenome KO screen to identify modifiers of aS seeding using an aS biosensor cell line. aS seeding spans many cellular processes that include endocytic uptake, vesicular trafficking, templated aggregate conversion, and protein degradation by both the proteasome and autophagy. Our screen identified proteins associated with vesicular trafficking between the ER, golgi and endosome, and the cellular stress response. Notably these pathways have been identified as modifiers of aS toxicity and stability in yeast and cell models ${ }^{31,32}$. Vesicular trafficking may have been particularly enriched since our screen and the aS biosensor cell line required that aS PFFs be applied with the carrier, Lipofectamine, as a means of facilitating endocytic uptake.

Our further studies expanded upon the role of VCP in aS seeding. VCP is a multifunctional protein necessary for many ubiquitin dependent processes that include protein degradation, vesicle trafficking, cell division and organelle clearance ${ }^{7}$. Recently, we identified a role for VCP in the recognition of permeabilized late endosomes and their subsequent lysophagic degradation ${ }^{15}$. Endocytosed proteopathic seeds such as aS and Tau enter the cytoplasm, where templated aggregate conversion of soluble monomer occurs, by damaging the endosomal membrane ${ }^{5,15,33}$. The fate of permeabilized late endosomes depends upon the degree of membrane damage. For example, some damaged endosomes are rapidly repaired by ESCRT proteins ${ }^{34}$. In contrast, endosomes damaged beyond repair are tagged by intracellular galectins such as galectin- $3^{15}$. Galectin positive endosomes recruit the ubiquitin ligase, Trim16 which ubiquitinates endosomal membrane proteins ${ }^{35}$. Notably, only lysine-63 linked ubiquitin chains on the endosome surface are targeted for lysophagy ${ }^{15}$. VCP in 
association with UBXD1, PLAA and the deubiquitinase YOD1 recognize and cleave lysine-48 linked ubiquitin chains on damaged late endosomes leaving lysine-63 linked ubiquitin chains allowing lysophagic degradation ${ }^{15}$. Loss of VCP or VCP disease mutant expression leads to the persistence of galectin-3 positive damaged late endosomes in cells, mouse models and patient tissue ${ }^{15,17}$.

A previous genomic screen using a Tau biosensor line identified several components of the ESCRT machinery as suppressors of Tau seeding ${ }^{36}$. Our screen identified VCP and Trim16 as suppressors of aS seeding. Further experiments found that knockdown of the VCP adaptor, UBXD1 that is necessary for lysophagy and VCP disease mutations defective in lysophagy also increase aS seeding. One distinction between these two screens is the use of lipofectamine to facilitate entry into the endocytic pathway of aS. Lipofectamine is known to damage endosomal membranes and may allow aS to generate larger "holes" that are not repaired by ESCRTS ${ }^{34}$. However, VCP's role in seeding was not exclusively lipofectamine dependent since VCP inhibition, knockdown or VCP mutant expression facilitated "naked" aS PFF seeding in HNs and in vivo.

VCP disease mutations cause multisystem proteinopathy (MSP) ${ }^{37}$. MSP is a late onset degenerative disorder with varied phenotypes and pathologies. These include inclusion body myopathy, ALS and FTD ${ }^{18}$. While the predominant aggregate pathology in the brain is reported to be TDP-43, several studies support the identification of aS positive aggregates in the brain ${ }^{8-}$ 10. Indeed, $\sim 5 \%$ of MSP patients have coincident Parkinsonism ${ }^{19}$. Notably, two recently identified families with a VCP-D395G mutation were found to have distinctive tau pathology leading to the description of a vacuolar tauopathy in the CNS ${ }^{11}$. Aggregate pathology in the skeletal muscle can be varied and include TDP-43, hnRNPA1/A2B1, SQSTM1, $\beta$-amyloid, 
desmin and VCP ${ }^{38-40}$. Weakness typically precedes the onset of neurodegenerative features such as dementia by 10 years suggesting that pathology begins in peripheral tissue such as skeletal muscle ${ }^{19}$. Whether protein aggregates from skeletal can seed the aggregate process in motor neurons or cortical neurons remain speculative. It is noteworthy that mice carrying the D395G missense mutation in VCP had an increase in Tau seeding supporting that VCP disease mutations can facilitate the propagation of different aggregate species ${ }^{11}$.

TDP-43 inclusions are a prominent feature in affected VCP disease tissue ${ }^{9,38}$. Our data further supports that TDP-43 PFF similar to aS-PFF can seed pathologic TDP-43 inclusions in HNs. TDP-43 seeding in HNs recapitulates several features of TDP-43 pathology such as phosphorylation, cytoplasmic redistribution and co-localization with stress granule markers and SQSTM1 ${ }^{41}$. VCP disease mutation expression increases pathologic TDP-43 inclusions as measured by an increase in phospho-TDP-43 immunostaining in HNs. This process is TDP-43 PFF dependent since monomeric TDP-43 fails to have the same effect. Whether the accumulation of TDP-43 inclusions is also affected by an additional role for VCP in stress granule clearance remains to be determined ${ }^{42}$. 


\section{METHOD AND MATERIAL}

\section{aS FRET seeding assay}

Generally, HEK 293T aS-CFP/YFP is plated in a black-bottomed 96-well plate with the density of $80 \mathrm{k} /$ well in DMEM media with $10 \%$ FBS and Penicillin-Streptomycin. Three control cell lines no-transfected HEK293T cells, aS-CFP, and aS-YFP transduced cells are cultured in the same condition. aS PFF is sonicated and prepared with OPTIMEN and 1ul Lipofectamine 20000 (Invitrogen) for each well and add dropwise to the cell after 48 hours. The cells are harvested after 24 hours for flow cytometry, same as reported. Briefly, the cells are detached by $0.05 \%$ trypsin-EDTA (Gibco), centrifuged, then fixed with 2\% PFA for 15 minutes, and finally resuspended in MACSima Running Buffer. The samples are analyzed by MACSQuant® VYB. FRET signal is excited by $405 \mathrm{~nm}$ lasers and detected by $525 / 50$ band pass filter. While the CFP and YFP are excited by $405 \mathrm{~nm}$ and $488 \mathrm{~nm}$ lasers and filtered by $450 / 50 \mathrm{~nm}$ and $525 / 50 \mathrm{~nm}$, respectively. The data is analyzed with FlowJ v10 software. Each FRET signal is calculated as percentage of FRET-positive cell timing Median FRET fluorescence intensity, and then normalized to its vehicle control.

VCP mcherry vectors are a gift from Hemmo Meyer's lab. The mutations is confirmed by Sanger sequencing (GENEWIZ) with VCP plasmid primers described before. 250ng of the plasmid is transfected with OPTIMEN and 0.5ul Fugene 6 (Promega) in each well 24 hours after plating. The cells then treated the same way as described above. The mcherry signal is excited by $561 \mathrm{~nm}$ laser and filtered via $615 / 20 \mathrm{~nm}$. The FRET signal is analyzed separately for mcherry positive and negative cells. 
Knockdown is achieved by reverse transfection of Human SMARTPOOL siRNA from

Dharmacon or Thermo Silencer Select siRNA. 6pmol siRNA is prepared in OPTIMEN and 0.3ul Lipofectamine $^{\mathrm{TM}}$ RNAiMAX (Invitrogen) according to its protocol and add to each well in a 96well plate. Then $80 \mathrm{k}$ suspended aS-CFP/YFP cell is plated in each well already with siRNA droplet. The aS PFF is treated 48 hours after plating as described above.

\section{Genome-wide CRISPR-Cas9 screens on aS biosensor line}

aS-CFP/YFP HEK293T cells were first transduced with WT cas9-blast.Single clone were sorted and cultured. The new cas 9 aS CFP/YFP line maintained the both aS-CFP and aS-YFP, and capable of seeding. Cas9 function were validated by a synethic gRNA and obtained with $99 \%$ NHEJ activity. About 50 million HEK293T syn CFP/YFP Cas9-blast cell were plated and then infected with pooled lentivirus with Burnello gRNA library (Addgene \#73178-LV) with 8ug/ml polybrene $(\mathrm{MOl}=0.3)$ the next day. After 24 hours, cells would undergo $1 \mathrm{ug} / \mathrm{ml}$ puromycin selection. Selected cells were replated at density of $6.4^{*} 10^{\wedge} 5$ cell $/ \mathrm{ml}$ after 96 hours of selection and replaced with fresh puromycin. 2 days later, harvest 1/5 of the cell ( 20 million) (untreated group, for library representation) and seeded the rest with $10 \mathrm{nM}$ aS -PFF. The seeded cells were collected the same as normal aS FRET assay as described above after 24 hours and sorted by Sony SY3200 cell sorter. DNA extraction were performed via QIAamp DNA Blood Midi on FRET positive and negative cells, as well as unsorted cells were separately, amplified by PCR, and deep sequenced by Illumina NovaSeq. The FRET positive and negative groups were compared with untreated total population group separately via Megack RRA. For pathway enrichment, 153 hits were input to g:profiler and plot via cytoscape as described before.

\section{aS and TDP-43 Fibril preparation}


aS PFF and monomer is generated as described before ${ }^{21}$. Briefly, purified recombinant aS monomer (2 mg/ml) was incubated in $20 \mathrm{mM}$ Tris- $\mathrm{HCl}, \mathrm{pH} 8.0,100 \mathrm{mM} \mathrm{NaCl}$ for $72 \mathrm{~h}$ at $37^{\circ} \mathrm{C}$ with shaking at $1000 \mathrm{rpm}$ in an Eppendorf Thermomixer. To determine the concentration of fibrils, the fibril reaction mix was centrifuged at $18,000 \times \mathrm{g}$ for $15 \mathrm{~min}$ to separate fibrils from monomer. The concentration of aS monomer in the supernatant was determined in a BCA protein assay according to the manufacturer's instructions, using a bovine serum albumin (BSA) standard curve. The measured decrease in aS monomer concentration was used to determine the concentration of fibrils in the $72 \mathrm{~h}$ fibril reaction mixture. To isolate pre-formed fibrils (PFF) from monomer, centrifuge the aS mix at $18,000 \times \mathrm{g}$ for $15 \mathrm{~min}$ to separate fibrils from monomer. Resuspend fibril pellet in the buffer containing $20 \mathrm{mM}$ Tris- $\mathrm{HCl}, 100 \mathrm{mM} \mathrm{NaCl}$, pH 8.0. aS PFF always freshly sonicated right before seeding.

Fluorescently labeled fibrils of aS were generated as previously described ${ }^{43}$. aS (1 mg/mL) was dissolved in $100 \mathrm{mM} \mathrm{NaHCO}$, sonicated for $15 \mathrm{~min}$, and spun through a $50 \mathrm{kD}$ filter (Amicon UFC5050) at 16,100 $\times \mathrm{g}$ for $15 \mathrm{~min}$. Alexa Fluor 647 NHS Ester (Thermo Fisher A20006) was dissolved in DMSO to $10 \mathrm{mg} / \mathrm{ml}$. Dye solution (molar ratio of dye: $\mathrm{aS}=2.1: 1$ ) was pipetted into monomerized aS during stirring and the mixture was stirred on bench for $\sim 1 \mathrm{~h}$. The mixture was then loaded onto a size exclusion column (Superdex 75 10/300 GL) and eluted with $5 \mathrm{mM} \mathrm{NaOH}$. The peak containing monomeric, labelled aS was collected, aliquoted and kept frozen until use. For aggregation assays, aS was dissolved in $10 \mathrm{mM} \mathrm{NaOH}$ at $1 \mathrm{mg} / \mathrm{mL}$. aS-647 was added at $5 \%$ labelling ratio. Then the solution was sonicated for 20 minutes, filtered through a $100 \mathrm{kD}$ membrane filter (Amicon Ultra, 540655) at $16,100 \times \mathrm{g}$ for $15 \mathrm{~min}$ at $4^{\circ} \mathrm{C}$. The protein and dye concentrations were measured by absorption at $280 \mathrm{~nm}$ and $647 \mathrm{~nm}$, respectively, and the labelling ratio was determined to be $4.9 \%$. To prepare labelled aS fibrils, monomer solution (5\% a-syn-AF647) and solutions were incubated in $100 \mathrm{mM} \mathrm{NaP}, \mathrm{pH} 7.4,10 \mathrm{mM} \mathrm{NaCl}$ for $120 \mathrm{~h}$ aggregated in a non-binding 96 well plate (Corning, \#3651) at a concentration of $30 \mu \mathrm{M}$ with 
intermittent shaking in aggregation buffer (100 mM NaP, pH 7.4, $10 \mathrm{mM} \mathrm{NaCl}$ ). A 2 mm diameter glass bead was added to each well to accelerate the aggregation through stirring. The plate was kept at $37^{\circ} \mathrm{C}$ and agitated by orbital shaking once every 1 minute for 5 seconds.

Recombinant TDP-43 (rTDP-43) was generated in Escherichia coli and purified as previously described. Briefly, rTDP-43 was bound to nickel-nitrilotriacetic acid-agarose and washed with wash buffer 1 (50 mM Tris, pH 8.0, $500 \mathrm{mM} \mathrm{NaCl}, 10 \%$ glycerol, 10\% sucrose, $1 \mathrm{mM}$ TCEP), washed with wash buffer 2 (50 mM Tris, pH 8.0, $500 \mathrm{mM} \mathrm{NaCl}, 10 \%$ glycerol, $10 \%$ sucrose, 50 $\mathrm{mM}$ Ultrol Grade imidazole, $\mathrm{pH}$ 8.0, $1 \mathrm{mM}$ TCEP), and finally eluted (50 mM Tris, $\mathrm{pH}$ 8.0, 500 $\mathrm{mM} \mathrm{NaCl}, 10 \%$ glycerol, $10 \%$ sucrose, $300 \mathrm{mM}$ Ultrol Grade imidazole, $\mathrm{pH}$ 8.0, $1 \mathrm{mM}$ TCEP). Then, rTDP-43 was ultracentrifuged in a Beckman Coulter Optima MAX-XP Ultracentrifuge at $40,000 \mathrm{rpm}$ for $30 \mathrm{~min}$ at $4^{\circ} \mathrm{C}$ to remove any pre-existing aggregates. Soluble protein was diluted to $4 \mathrm{uM}$ in the reaction buffer (50 mM Tris, $\mathrm{pH} 8.0,250 \mathrm{mM} \mathrm{NaCl}, 5 \%$ glycerol, $5 \%$ sucrose, $150 \mathrm{mM}$ Ultrol Grade imidazole, pH 8.0, 0.5mM TCEP). rTDP-43 aggregation was started by shaking at $1,000 \mathrm{rpm}$ at $22^{\circ} \mathrm{C}$ for 30 min with an Eppendorf ThermoMixer $\mathrm{C}$. Samples were incubated at $22^{\circ} \mathrm{C}$ and collected after one to ten days. Full length TDP-43 recombinant protein is produced as previously described ${ }^{21}$. To obtain TDP-43 monomer, TDP43 protein was ultracentrifuge $40,000 \mathrm{~g} 30$ mins at $4^{\circ} \mathrm{C}$. The supernatant was collected and freshly used.

\section{Primary neuronal culture}

WT hippocampal neurons were obtained from E17-18 mice (Charles River). Hippocampi are dissected in calcium- and magnesium-free Hanks' Balanced Salt solution (HBSS) and dissociated by $0.05 \%$ Trypsin-EDTA at $37 \mathrm{C}$ for $5-10$ mins and followed by $1 \%$ DNase I for 2 mins ${ }^{44}$. The cells are then resuspended with plating media to the concentration of $125 \mathrm{k} / \mathrm{ml}$ and 
plated on Poly-D-lysine coated plates or coverslips. The media is changed to neurobasal media (neurobasal plus $+B 27+5 \mathrm{mM} \mathrm{L-Glutamine)} \mathrm{after} \mathrm{2-4} \mathrm{hours.} 1 \mathrm{mM}$ Ara-C is added to inhibit the growth of glia. The aS PFF is sonicated and add directly to the cell at DIV10.

VCP and UBXD1 shRNA is delivered via lentivirus. Lentivirus were added to the neurons at DIV5 with $\mathrm{MOI} \geq 5$. For LLoMe or VCP inhibitors experiments, drug or vehicle control was added at the same time with aS PFF for 4 hours, and then the media is fully exchanged to the conditioned media without drug and aS. The neurons were harvested at DIV15.

R155H/WT neurons were cultured from embryos from R155H/WT intercross. The hippocampus from each embryo was dissected and cultured separately and then plate at the same density. The genotypes were examined by PCR (Transnetyx) (Forward Primer:

CCTCTAATTGCACTTGTATTGCTTTGT; Reverse Primer:

CTGGGATCTGTCTCTACAACTTTGA).

\section{Immunohistochemistry}

Cells were fixed in 4\% PFA for 10 mins and permeabilized with $0.1 \%$ Triton X-100 in PBS for 10 mins. Then the cells were blocked with $2 \%$ BSA in PBS at RT for 1 hour. Cells were stained with primary antibody at $4{ }^{\circ} \mathrm{C}$ overnight, followed by 3 washes with PBS. Cells were then incubated with the Alexa 488555 or 647 tagged secondary antibody in 1:500 dilution for 1 hour at RT. The nucleus was stained with DAPI (1:1000) for 10 min at RT. After 3 wash with PBS, the cells are mounted by Mowiol. Pictures were taken by either Nikon Eclipse 80i fluorescence microscope or a Hamamatsu NanoZoomer. The images were process via ImageJ or NDP.view2. The antibody is listed in Appendix.

\section{Immunoblot}


Mouse cortex or cells are lysed in RIPA buffer with protease inhibitor cocktails (PMSF and PIC) followed by two $30 \mathrm{sec}$ on and 30 off sonication cycle at $50 \%$ power. The protein concentration is normalized by the BCA assay. Samples were loaded into 10 to $15 \%$ gel and transferred into nitrocellulose or PVDF membrane. The membranes were blocked by $5 \%$ milk in PBS- $0.2 \%$ Tween20 and incubated with primary antibody in blocking solution overnight at $4{ }^{\circ} \mathrm{C}$ degree. Membrane then were washed three time with PBS-0.2\% Tween20, and incubated with secondary goat anti- rabbit, mouse HRP antibody (1:5000) for 1 hour. Blot were rinsed three time with PBS- $0.2 \%$ Tween 20 and probed by fresh mixture of ECL reagents at dark and then exposed by SYNGENE.

To fractionate insoluble portion of aS, we preformed sequential extraction as described ${ }^{45}$. Briefly, neurons were first dissolved in TBS-1\%Tx-100, and sonicated for 10 cycles of 30 s on, 30 s off with $50 \%$ power. The lysate would incubate on ice for 30 mins. $1 / 10$ of the lysate were saved as total protein, while the remaint was ultracentrifuged $100,000 \mathrm{~g} 4{ }^{\circ} \mathrm{C}$ for $30 \mathrm{mins}$. The supernant was collected as Tx- 100 soluble fraction.the pellet was wash with TBS-1\%Tx-100, sonicated and ultracnetrifuged. Ultimately, the pellet was resupsended with TBS-2\%SDS, and sonicated for 15 cycles of $30 \mathrm{~s}$ on, $30 \mathrm{~s}$ off. Soluble and insoluble fraction were run by western blot as normal. The loading amount were determined by the concentration of total protein measured by BCA.

TPD-43 soluble and insoluble extraction is done as our previous method. Briefly, neurons from one 6 well were first lysed with RIPA buffer with protease inhibitor cocktails (RIPA buffer) on ice. The lysate then sonicated with QSONICA sonicator for 10 cycles of 30 s on, 30 s off with $50 \%$ power. $1 / 10$ of the lysate were saved as total protein. The rest was ultracentrifuged at $100,000 \mathrm{~g}$ $4^{\circ} \mathrm{C}$ for 30 mins. The supernatant was kept as RIPA soluble fraction. The pellet was then wash with RIPA buffer once, resonicated and ultracentrifuged with the same condition. The pellet 
finally resupsend with same amount of UREA buffer (30 mM Tris, $\mathrm{pH} 8.8,7 \mathrm{M}$ urea, 2M thiourea, and 4\% CHAPS) as insoluble fraction. Soluble and insoluble fraction were run by western blot as normal. The loading amount were determined by the concentration of total protein measured by BCA.

\section{Intrastriatal injection and mouse brain harvest}

Both mice are Intraperitoneal injected with $75 \mathrm{mg}$ tamoxifen $/ \mathrm{kg}$ body weight at two months of age and wait one month for gene knockdown. The existence of the R155C mutation allele and VCP flop knockdown confirmed by PCR. The primers are listed in Appendix. Intrastriatial injection is performed as described. aS PFF is prepared as described above and diluted in sterile PBS. aS PFF is sonicated 10 mins before injection. The mouse is anesthetized and injected at the dorsal striatum (Bregma $=0.2 \mathrm{~mm}$, midline $=2.0 \mathrm{~mm}$, depth $=-3.2 \mathrm{~mm}$ ) of the left hemisphere. The same amount of PBS is used as vehicle control. The recovery of mice is monitored in the following week and sacrificed after 90 days. Mouse was first anesthetized in Isofluorane chamber and perfused with PBS containing herapin. The whole brains were removed from the skull and fixed in $4 \%$ PFA overnight at $4{ }^{\circ} \mathrm{C}$ degree and cut coronally into 40micrometer sections for immunostaining.

\section{Statistical Analysis}

The data (except CRISPR screening) is analyzed by GraphPad Prism 9. Statistical tests included unpaired t test, one-way ANOVA, multiple t-test, simple linear regression, nonlinear regression and two-way ANOVA. Data were displayed as mean \pm SEM. Two-stage step-up method of benjamini krieger and yekutieli, Dunnett, sidak correction were used to minimize false alarm from multiple comparsion. 
Figure Legends:

Figure 1: Genome-wide CRISPR/Cas9 screen identifies genes protective to aS seeding.

(A) aS biosensor line stably expressing spCAS9 was transduced with sgRNA lentiviral library

(Brunello). Following antibiotic selection, biosensors were seeded with aS PFF and flow sorted 24 hours later. Genomic DNA from positive and negative groups as well as unsorted total population were collected and decoded by NGS. (B) Volcano plot of genes identified in the screen. Colored in blue are all the genes plotted from FRET-group, while colored in black are those plotted from FRET+. Red dots are protective hits from both groups (5\% FDR). Those genes with a fold change $<0.5$ were underrepresented in FRET- cells and genes $>2$ were overrepresented in the FRET+ cells. (C) Pathway analysis of 153 protective genes via g:profiler. The enriched pathway is visualized by cytoscape. (D) Normalized FRET of siRNA knockdown and aS PFF treated biosensors using 9 protective genes identified in the screen. (n $\geq 9$ repeats ; ${ }^{* *} p<0.001,{ }^{* *} p<0.01$ and ${ }^{*} p<0.05$ by one-way ANOVA. error bars are \pm S.E.M.) (E) Immunoblot with VCP and GAPDH of cell lysates from aS biosensors/spCas9 cells treated with a VCP gRNA (F) Normalized FRET of cells in (E) treated with 24-hour aS PFF. ( $P=0.0439$ by a two-tailed t-test). $n=4$ biologically independent FRET assay. Data is mean \pm S.E.M.

Figure 2: VCP inhibition enhances aS seeding independent of uptake or changes in aS protein levels.

(A) aS biosensors were treated with scrambled control siRNA or VCP siRNA for 48 hours prior the application of aS monomer or PFF with or without Lipofectamine and measured for FRET efficiency at 24 or 48 hours. (B) Normalized FRET of aS biosensors co-treated with aS monomer or PFF with the indicated chemical compound or vehicle for four hours followed by 
washout. In the case of CB-5083 and Eer1, aS biosensors were treated for 24 hours. ( $n \geq 9$ repeats for each PFF treated conditions; n.s. for all monomer treated pairs; ${ }^{* \star * *} p<0.0001$ and ${ }^{\star \star *} p<0.001$ by two-way ANOVA in some PFF treated pairs as indicated. error bars are \pm S.E.M.) (C) 647 fluorescent intensity (seed uptake) from aS biosensors co-treated with Alexa647 tagged aS PFF and vehicle or LLoME for 4 hours. $n=3$ biological repeat. Data is mean \pm s.e.m. (D) 647 fluorescent intensity (seed uptake) from aS biosensors co-treated with Alexa-647 tagged aS PFF and vehicle or NMS-873 for 4 hours. $n=3$ biological repeat. Data is mean \pm s.e.m. (E) 647 fluorescent intensity (seed uptake) of aS biosensors treated with scrambled or VCP siRNA for 48 hours and then Alexa-647 tagged aS PFF for four hours. $n=4$ biological repeat. Data is mean \pm s.e.m. (F) 647 fluorescent intensity (seed uptake) from aS biosensors co-treated with Alexa-647 tagged aS PFF and Dynogo-4a or DMSO for four hours and measured after 4 or 24 hours. $n=4$ biological repeat. Data is mean \pm s.e.m. (G) CFP fluorescent intensity in aS biosensors following vehicle or LLoME for 24 hours. $n=3$ biological repeat. Data is mean \pm s.e.m $(\mathrm{H})$ CFP fluorescent intensity in aS biosensors following vehicle or NMS-873 for 24 hours. $n=3$ biological repeat. Data is mean \pm s.e.m (I) CFP fluorescent intensity in aS biosensors following scrambled or VCP siRNA treatment for 48 hours. $n=4$ biological repeat. Data is mean \pm s.e.m $(\mathrm{J})$ Immunoblot of total a-synuclein (syn-1), FK-2, tubulin or GAPDH from aS biosensors treated with DMSO or VCP inhibitor NMS-873 for 4 hours. (K) Immunoblot of VCP, 14-3-3, total a-synuclein (syn-1) or HSP90 from aS biosensors treated with scrambled or VCP siRNA for 48 and 72 hours. (L) Normalized FRET of aS biosensors co-treated with aS monomer or PFF with the indicated chemical compound or vehicle for 24 hours. $(n=9$ repeats for each PFF treated conditions; n.s. for all monomer treated pairs; adjusted $p=0.0470$ and $\mathrm{p}=0.0124$ for DTT and Tunicamycin by two-way ANOVA. Error bars are \pm S.E.M.)

Figure 3: VCP inhibition enhances aS seeding in neurons. 
(A) Immunoblot for aS from detergent soluble and insoluble lysates of HNs treated for 4 hours with aS monomer or PFF and then harvested 5 days later. Note that the $2 \%$ SDS insoluble fraction has high molecular weight aS positive multimers. GAPDH is the loading control. (B) Immunofluorescence for phospho-aS and Tuj1 (neurite marker) in HNs co-treated with aS PFF and LLoMe $(1 \mathrm{uM})$ or vehicle for 4 hours. Immunostaining performed after 5 days. Scale bar $=50$ and $10 \mu \mathrm{m}$ for original and zoom-in figure respectively (C) Quantitation of phospho-aS/Tuj1 staining as in 3B $\left({ }^{* * *}\right.$ Multiple fields were captured in each conditions and quantified independently, $n=37,38$ for ethanol and LLoMe groups respectively. Experiments were repeated from 3 different cultures. ${ }^{* \star *} p<0.0001$ by student's t-test. Error bars are \pm S.E.M.). (D) Immunofluorescence for phospho-aS and Tuj1 in HNs co-treated with aS PFF and ML240 $(100 \mathrm{nM})$ or DMSO control for 4 hours. Scale bar $=50$ and $10 \mu \mathrm{m}$ for original and zoom-in figure respectively (E) Quantitation of phospho-aS/Tuj1 staining as in 3D (Multiple fields were captured in each conditions and quantified independently, $n=32$ for DMSO and ML240 group. Experiments were repeated from 3 different cultures. ${ }^{* *} p<0.01$ by student's t-test. Error bars are \pm S.E.M.). (F) Immunofluorescence for phospho-aS and Tuj1 in HNs infected with lentiviral vectors expressing scrambled or one of two different shRNAs targeting VCP for 2 days and then treated with aS PFF for 5 days. Scale bar $=50$ and $10 \mu \mathrm{m}$ for original and zoom-in figure respectively (G) Quantitation of phospho-aS/Tuj1 staining as in 3F (Multiple fields were captured in each conditions and quantified independently, $n=29,27$ and 9 for scramble, VCP shRNA groups. Experiments were repeated from 3 different cultures. ${ }^{* *} p<0.01$, ${ }^{* * *} p<0.001$ by oneway ANOVA. Error bars are \pm S.E.M.).

Figure 4: VCP disease mutations increase aS seeding.

(A) aS biosensors were treated with scrambled control siRNA, siRNAs targeting the indicated genes for 48 hours prior the application of aS PFF and harvested for FRET efficiency at 24 
hours. $n \geq 11$ repeats for each KD. Adjusted $p=0.0147$ and $p<0.0001$ for UBXD1 and VCP KD respectively by one-way ANOVA. Error bars are \pm S.E.M.) (B) Immunofluorescence for phospho-aS and Tuj1 in HNs infected with lentiviral vectors expressing scrambled or a shRNA targeting UBXD1 for 5 days and then treated with aS PFF for 5 days. (C) Quantitation of phospho-aS/Tuj1 staining as in 2B ( $\left.{ }^{* * *} \mathrm{P}<0.0001\right)$. (D) aS biosensors were transfected with plasmids expressing VCP-WT, or one of three disease mutations (R95G, R155H and A232E) fused to an mcherry tag for 24 hours and then treated with aS PFF. FRET efficiency is quantified in mCherry+ and mCherry- cells separately and all normalized by VCP ${ }^{W T}$. $(n=11$ repeats for each group. ${ }^{* * *} \mathrm{p}<0.001 ;{ }^{* * *} \mathrm{p}<0.0001$; ns, no significance; two-way ANOVA with Dunnett's correction) (E) Immunofluorescence for phospho-aS and Tuj1 in HNs form wild-type mice or mice carrying a VCP-R155H knockin allele $\left(\mathrm{VCP}^{\mathrm{R} 155 \mathrm{H} / \mathrm{WT}}\right)$ treated with aS PFF for 5 days. (F) Quantitation of phospho-aS/Tuj1 staining as in 4C (Multiple fields were captured in each conditions and quantified independently. Neurons coming from 7 and 12 independent cultures from WT and VCP ${ }^{R 155 H / W T}$ embryos. Outlier is removed by ROUT method, $\mathrm{Q}=1 \%$, followed by Student's t test. $p<0.0001$ )

Figure 5: VCP disease mutant mice accumulate Galectin-3.

(A) Representative immunoblot for VCP, p62, 14-3-3, BiP/GRP78, LC3, Lamp1, Galectin-3, HSP90, and ubiquitin (FK2) from cortical brain lysates of 4 month old C57 (n=3), VCP ${ }^{R 155 H / W T}$ $(n=4)$ and cVCP-R155C ( $n=4)$ mice. In the case of cVCP-R155C mice, they are intraperitoneally injected with tamoxifen at 90 days of age and the brain was collected after one month. (B) Quantification of band intensities of VCP, p62, Bip/GRP78, LC3, FK2, Lamp1 and Gal3. (C) Representative immunoblot for VCP, p62, 14-3-3, Lamp1, Galectin-3, and ubiquitin (FK2) from cortical brain lysates of cVCP-R155C mice following one month or six months of i.p. tamoxifen 
treatment. (D) Quantitation of band intensities of VCP, p62, Lamp1, FK2 and Gal3 ( $\mathrm{n}=3$ for both groups).

Figure 6: VCP disease mutations enhance aS seeding in vivo.

(A) Schematic of coronal sections through mouse brains. Shaded regions indicate areas utilized for quantitation and boxes denote regions corresponding to images in B and D. Red dots represent p-syn detected. (B) Representative immunofluorescence images with pSer129-syn antibody of anterior cortices from C57, cVCP-R155C, and VCP $\mathrm{P}^{\mathrm{R} 155 \mathrm{H} / \mathrm{WT}}$ mice injected unilaterally into the striatum with 5 ug aS PFF after 90 days. 4 month aged C57 ( $n=10)$, VCP R155H/WT $(n=5)$ and VCP R155C/FL; Rosa26-Cre ERT2 (cVCP-R155C) (n=5). Scale bar=25 um. (C) Quantitation of the percentage of $p$-syn in entire anterior cortices ( ${ }^{*} \mathrm{P}<0.05$ by student's t-test).

(D) Representative immunofluorescence images with pSer129-syn antibody of posterior cortices from C57, cVCP-R155C, and VCP ${ }^{R 155 H / W T}$ mice as described in A. scale bar=25 $\mu \mathrm{m}$. (E) Quantitation of the percentage of $p$-syn in posterior cortices $\left({ }^{*} P<0.05\right.$, ${ }^{* *} P<0.01\left({ }^{*} P<0.05\right.$ by student's t-test). (F-G) Quantitation of p-syn in Amygdala and substantia Nigra. (ns, no significance student's t-test)

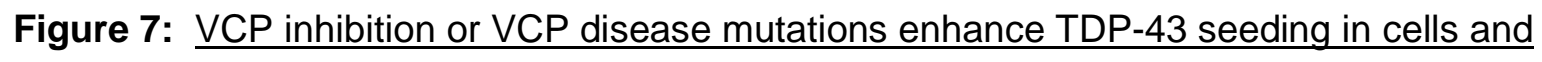
neurons.

(A) Immunofluorescent staining for pTDP-43 (red) and nuclei (blue) in primary hippocampal neurons treated with varied concentrations of TDP-43 PFF for 5 days. Scare bar $=50 \mu \mathrm{m}(\mathrm{B})$ Quantitation of area of pTDP-43 immunofluorescence as in 7D. $n \geq 5$ for each concentrations. (C) Quantitation of the ratio of nuclear to cytoplasmic fluorescence of pTDP-43 as in 7D. pTDP43 fluorescence intensity was measured separately in nucleus and cytoplasm. More than 45 nucleus are counted in each group. (D) Immunoblot for TDP-43 from detergent soluble and 
insoluble lysates of HNs treated with TDP-43 monomer or PFF and then harvested after 1 or 5 days. Note that the $2 \%$ SDS insoluble fraction has high molecular weight TDP-43 positive multimers. 14-3-3 is a loading control. (E) Immunofluorescent images for pTDP-43 (green), SQSTM1 (red upper panels), TIA1 (red lower panels) and DAPI nuclei (blue) from neurons treated with TDP-43 monomer or PFF for five days. Scare bar= 50 and $10 \mu \mathrm{m}$ for original and zoom-in images. (F) Immunofluorescence for phospho-TDP-43 (red) and Tuj1 (green) in HNs form wild-type mice or mice carrying a VCP-R155H knockin allele (VCPR155H/WT) treated with TDP-43 for PFF for 5 days. (Scare bar $=50$ and $10 \mu \mathrm{m}$ for original and zoom-in images). (G) Quantitation of phospho-TDP/Tuj1 staining as in $7 \mathrm{I}$ (Neurons coming from 3 and 4 independent cultures from WT and VCP ${ }^{\mathrm{R} 155 \mathrm{H} / \mathrm{WT}}$ embryos. Outlier is removed by ROUT method, $\mathrm{Q}=1 \%$, followed by Student's t test. $\mathrm{n}=26,21$ for WT and VCP ${ }^{\mathrm{R} 155 \mathrm{H} / \mathrm{WT}}$ group respectively. ${ }^{* * *}$ $P<0.0001$.)

Figure S1: spCas9-gRNA aS biosensor maintain normal seeding capacity. spCas9 aS biosensor was treated with pooled library and selected as described in Fig 1A, followed by aS seeding at different concentration. After 24hours, cells are harvested and the percentage of FRET is measured by flow cytometry.

Figure S2: $\underline{\text { aS biosensor shows seeding activities in a concentration dependent manner. }}$ (A) aS biosensor was treated with aS PFF at different concentration for 4 or 24 hours. Cells are harvested after 24 hours and analyzed the same as 2A. (B) Alexa647 tagged aS PFF show moderate seeding capacity according to FRET assay.

Figure S3: Monomer is insufficient to induce seeding under drug treatment or VCP KD. (A) Representative pictures of HNs treated with aS monomer for 4 hours together with either LLoMe 
(1uM), ML240 or vehicle cotnrols at DIV10. The cells are harvested after 5 days and stained with p-syn and Tuj1. aS PFF treated HNs are presented as positive control. (B) Representative pictures of HNs of VCP KDs or scramble control treated with aS monomer at DIV10. The cells are harvested after 5 days and stained with p-syn and Tuj1. aS PFF treated HNs are presented as positive control.

Figure S4: Gene knockdown efficiency and VCP vector transfection. (A) aS biosensor is reverse transfected with siRNA (6pmol). Cell lysis are saved at 48 and 72 hours after transfection. Western blot is preformed on each KD for protein level change. (B) aS biosensor cells with different KD are transduced with Alexa-647 tagged aS PFF for 4 hours. The percentage of uptake is calculated the same way as $2 F$. (C) aS biosensor VCP mutated vectors showed similar level of VCP exogenous expression, tested by Western blot.

Figure S5: Genotyping of cVCP-R155C mice. Cortical tissues from 4 month old cVCP-R155C and C57 are genotyped. cVCP-R155C mice are i.p. injected with Tamoxifen in 5 continuous day and harvested after one month for genotyping.

Figure S6: pTDP-43 is TDP-43 PFF specific in hippocampal neurons. TDP4-43 monomer, TDP-43 PFF or aS PFF is added as indicated. After 5 days, HNs are harvested and stained with (p)TDP-43 and (p)syn(81A) 


\section{REFERENCES}

1. Goedert, M. Alpha-synuclein and neurodegenerative diseases. Nat Rev Neurosci 2, 492-501 (2001).

2. Prusiner, S.B. et al. Evidence for alpha-synuclein prions causing multiple system atrophy in humans with parkinsonism. Proc Natl Acad Sci U S A 112, E5308-5317 (2015).

3. Brettschneider, J., Del Tredici, K., Lee, V.M. \& Trojanowski, J.Q. Spreading of pathology in neurodegenerative diseases: a focus on human studies. Nat Rev Neurosci 16, 109-120 (2015).

4. Yamasaki, T.R. et al. Parkinson's disease and multiple system atrophy have distinct alphasynuclein seed characteristics. J Biol Chem 294, 1045-1058 (2019).

5. Jiang, P., Gan, M., Yen, S.H., McLean, P.J. \& Dickson, D.W. Impaired endo-lysosomal membrane integrity accelerates the seeding progression of alpha-synuclein aggregates. Sci Rep 7, 7690 (2017).

6. Hollerhage, M., Bickle, M. \& Hoglinger, G.U. Unbiased Screens for Modifiers of Alpha-Synuclein Toxicity. Curr Neurol Neurosci Rep 19, 8 (2019).

7. Meyer, H. \& Weihl, C.C. The VCP/p97 system at a glance: connecting cellular function to disease pathogenesis. J Cell Sci 127, 3877-3883 (2014).

8. Spina, S. et al. Phenotypic variability in three families with valosin-containing protein mutation. Eur J Neurol 20, 251-258 (2013).

9. Neumann, M. et al. TDP-43 in the ubiquitin pathology of frontotemporal dementia with VCP gene mutations. J Neuropathol Exp Neurol 66, 152-157 (2007).

10. Forman, M.S. et al. Novel ubiquitin neuropathology in frontotemporal dementia with valosincontaining protein gene mutations. J Neuropathol Exp Neurol 65, 571-581 (2006).

11. Darwich, N.F. et al. Autosomal dominant VCP hypomorph mutation impairs disaggregation of PHF-tau. Science 370 (2020).

12. Ju, J.S., Miller, S.E., Hanson, P.I. \& Weihl, C.C. Impaired protein aggregate handling and clearance underlie the pathogenesis of p97/VCP-associated disease. J Biol Chem 283, 30289-30299 (2008).

13. Ritz, D. et al. Endolysosomal sorting of ubiquitylated caveolin-1 is regulated by VCP and UBXD1 and impaired by VCP disease mutations. Nat Cell Biol 13, 1116-1123 (2011).

14. Blythe, E.E., Gates, S.N., Deshaies, R.J. \& Martin, A. Multisystem Proteinopathy Mutations in VCP/p97 Increase NPLOC4.UFD1L Binding and Substrate Processing. Structure 27, 1820-1829 e1824 (2019).

15. Papadopoulos, C. et al. VCP/p97 cooperates with YOD1, UBXD1 and PLAA to drive clearance of ruptured lysosomes by autophagy. EMBO J 36, 135-150 (2017).

16. Zhang, T., Mishra, P., Hay, B.A., Chan, D. \& Guo, M. Valosin-containing protein (VCP/p97) inhibitors relieve Mitofusin-dependent mitochondrial defects due to VCP disease mutants. Elife 6 (2017).

17. Arhzaouy, K. et al. VCP maintains lysosomal homeostasis and TFEB activity in differentiated skeletal muscle. Autophagy 15, 1082-1099 (2019).

18. Taylor, J.P. Multisystem proteinopathy: intersecting genetics in muscle, bone, and brain degeneration. Neurology 85, 658-660 (2015).

19. Mehta, S.G. et al. Genotype-phenotype studies of VCP-associated inclusion body myopathy with Paget disease of bone and/or frontotemporal dementia. Clin Genet 83, 422-431 (2013).

20. Conicella, A.E. et al. TDP-43 alpha-helical structure tunes liquid-liquid phase separation and function. Proc Natl Acad Sci U S A 117, 5883-5894 (2020).

21. French, R.L. et al. Detection of TAR DNA-binding protein 43 (TDP-43) oligomers as initial intermediate species during aggregate formation. J Biol Chem 294, 6696-6709 (2019). 
22. Porta, S. et al. Patient-derived frontotemporal lobar degeneration brain extracts induce formation and spreading of TDP-43 pathology in vivo. Nat Commun 9, 4220 (2018).

23. Beilina, A. et al. The Parkinson's Disease Protein LRRK2 Interacts with the GARP Complex to Promote Retrograde Transport to the trans-Golgi Network. Cell Rep 31, 107614 (2020).

24. Mangieri, L.R. et al. ATP6VOC knockdown in neuroblastoma cells alters autophagy-lysosome pathway function and metabolism of proteins that accumulate in neurodegenerative disease. PLoS One 9, e93257 (2014).

25. Garcia-Esparcia, P. et al. Dementia with Lewy Bodies: Molecular Pathology in the Frontal Cortex in Typical and Rapidly Progressive Forms. Front Neurol 8, 89 (2017).

26. Colla, E. Linking the Endoplasmic Reticulum to Parkinson's Disease and Alpha-Synucleinopathy. Front Neurosci 13, 560 (2019).

27. Wojcik, C. et al. Valosin-containing protein (p97) is a regulator of endoplasmic reticulum stress and of the degradation of $\mathrm{N}$-end rule and ubiquitin-fusion degradation pathway substrates in mammalian cells. Mol Biol Cell 17, 4606-4618 (2006).

28. Chou, T.F. et al. Reversible inhibitor of $\mathrm{p} 97, \mathrm{DBeQ}$, impairs both ubiquitin-dependent and autophagic protein clearance pathways. Proc Natl Acad Sci U S A 108, 4834-4839 (2011).

29. Volpicelli-Daley, L.A. et al. Exogenous alpha-synuclein fibrils induce Lewy body pathology leading to synaptic dysfunction and neuron death. Neuron 72, 57-71 (2011).

30. Nalbandian, A. et al. A progressive translational mouse model of human valosin-containing protein disease: the VCP(R155H/+) mouse. Muscle Nerve 47, 260-270 (2013).

31. Cooper, A.A. et al. Alpha-synuclein blocks ER-Golgi traffic and Rab1 rescues neuron loss in Parkinson's models. Science 313, 324-328 (2006).

32. Rousseaux, M.W.C. et al. A Druggable Genome Screen Identifies Modifiers of alpha-Synuclein Levels via a Tiered Cross-Species Validation Approach. J Neurosci 38, 9286-9301 (2018).

33. Flavin, W.P. et al. Endocytic vesicle rupture is a conserved mechanism of cellular invasion by amyloid proteins. Acta Neuropathol 134, 629-653 (2017).

34. Skowyra, M.L., Schlesinger, P.H., Naismith, T.V. \& Hanson, P.I. Triggered recruitment of ESCRT machinery promotes endolysosomal repair. Science 360 (2018).

35. Chauhan, S. et al. TRIMs and Galectins Globally Cooperate and TRIM16 and Galectin-3 Co-direct Autophagy in Endomembrane Damage Homeostasis. Dev Cell 39, 13-27 (2016).

36. Chen, J.J. et al. Compromised function of the ESCRT pathway promotes endolysosomal escape of tau seeds and propagation of tau aggregation. J Biol Chem 294, 18952-18966 (2019).

37. Watts, G.D. et al. Inclusion body myopathy associated with Paget disease of bone and frontotemporal dementia is caused by mutant valosin-containing protein. Nat Genet 36, 377381 (2004).

38. Weihl, C.C. et al. TDP-43 accumulation in inclusion body myopathy muscle suggests a common pathogenic mechanism with frontotemporal dementia. J Neurol Neurosurg Psychiatry 79, 11861189 (2008).

39. Kim, H.J. et al. Mutations in prion-like domains in hnRNPA2B1 and hnRNPA1 cause multisystem proteinopathy and ALS. Nature 495, 467-473 (2013).

40. Weihl, C.C., Pestronk, A. \& Kimonis, V.E. Valosin-containing protein disease: inclusion body myopathy with Paget's disease of the bone and fronto-temporal dementia. Neuromuscul Disord 19, 308-315 (2009).

41. de Boer, E.M.J. et al. TDP-43 proteinopathies: a new wave of neurodegenerative diseases. J Neurol Neurosurg Psychiatry (2020).

42. Buchan, J.R., Kolaitis, R.M., Taylor, J.P. \& Parker, R. Eukaryotic stress granules are cleared by autophagy and Cdc48/VCP function. Cell 153, 1461-1474 (2013). 
43. Nahass, G.R. et al. Brazilin Removes Toxic Alpha-Synuclein and Seeding Competent Assemblies from Parkinson Brain by Altering Conformational Equilibrium. J Mol Biol 433, 166878 (2021).

44. Kaech, S. \& Banker, G. Culturing hippocampal neurons. Nat Protoc 1, 2406-2415 (2006).

45. Volpicelli-Daley, L.A., Luk, K.C. \& Lee, V.M. Addition of exogenous alpha-synuclein preformed fibrils to primary neuronal cultures to seed recruitment of endogenous alpha-synuclein to Lewy body and Lewy neurite-like aggregates. Nat Protoc 9, 2135-2146 (2014). 
bioRxiv preprint doi: https://doi.org/10.1101/2021.07.12.452081; this version posted July 13, 2021. The copyright holder for this preprint (which

Figure 1

A

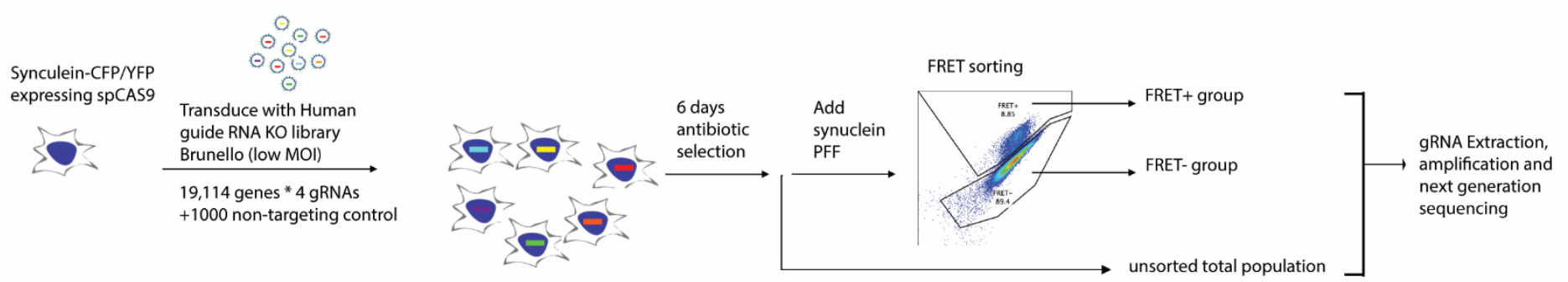

B

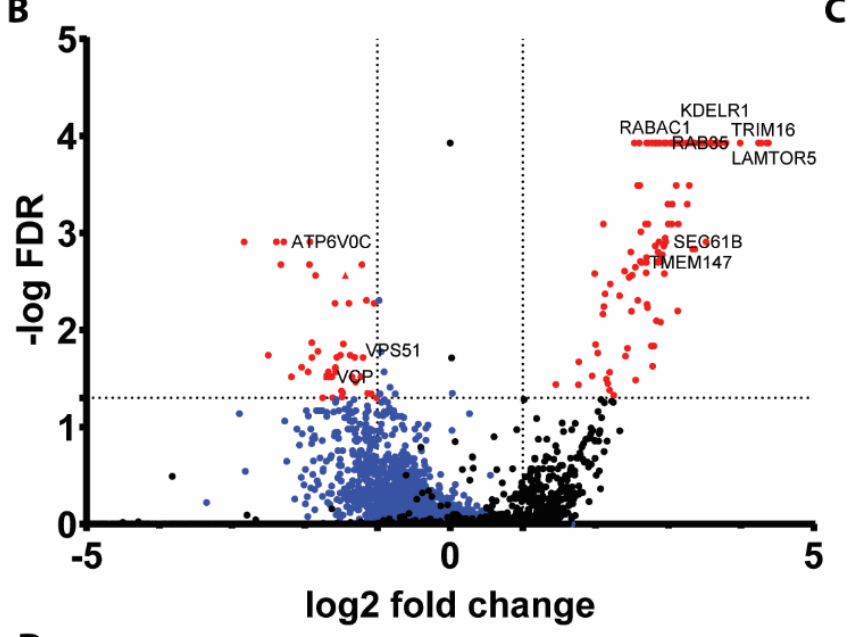

D

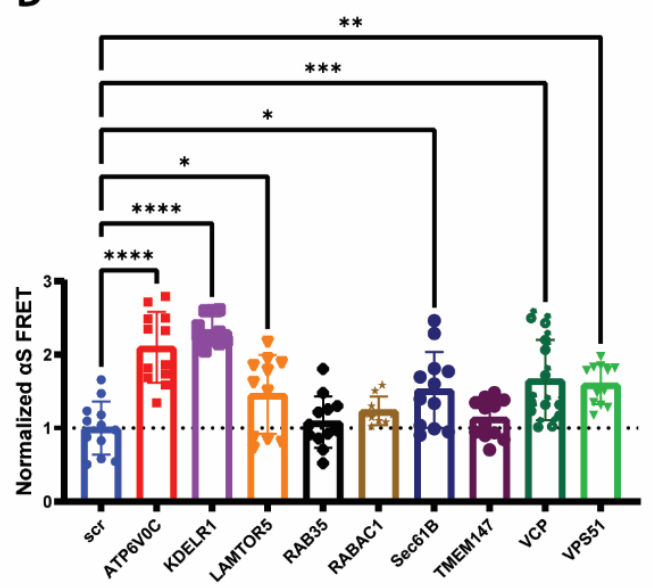

C

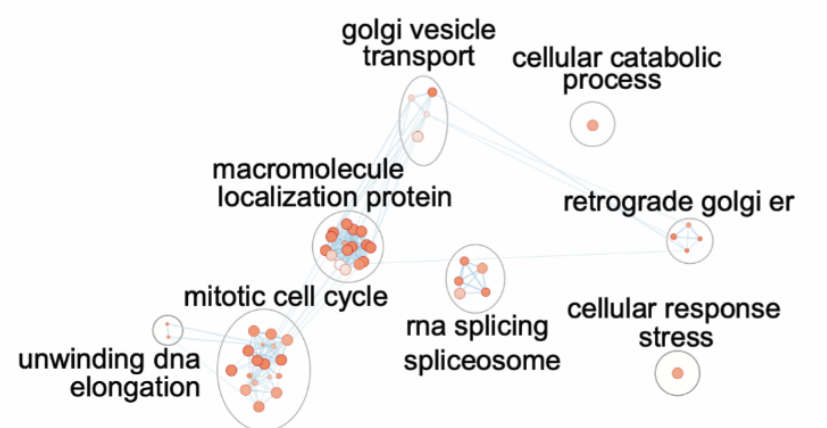

F

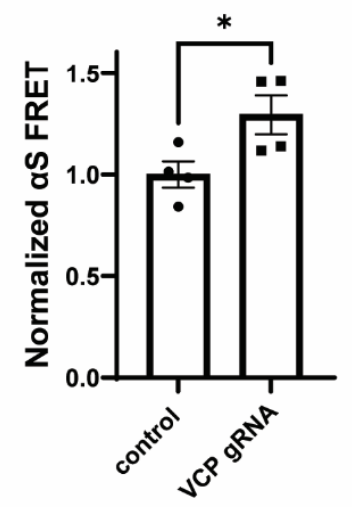


FigbioRxiv preprint doi: https://doi.org/10.1101/2021.07.12.452081; this version posted July 13, 2021. The copyright holder for this preprint (which Figure 2 was not certified by peer review) is the author/funder. All rights reserved. No reuse allowed without permission.

$$
\text { A }
$$

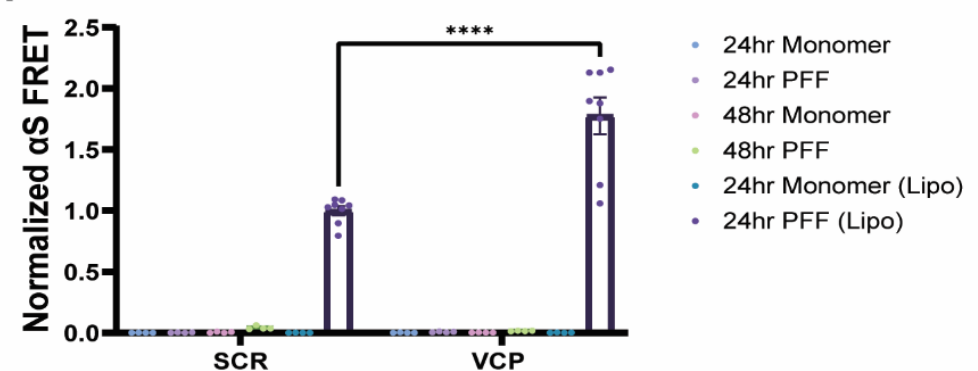

B

- Vehicle + Monomer

- Drug + Monomer

- Vehicle + aS PFF

Drug + aS PFF

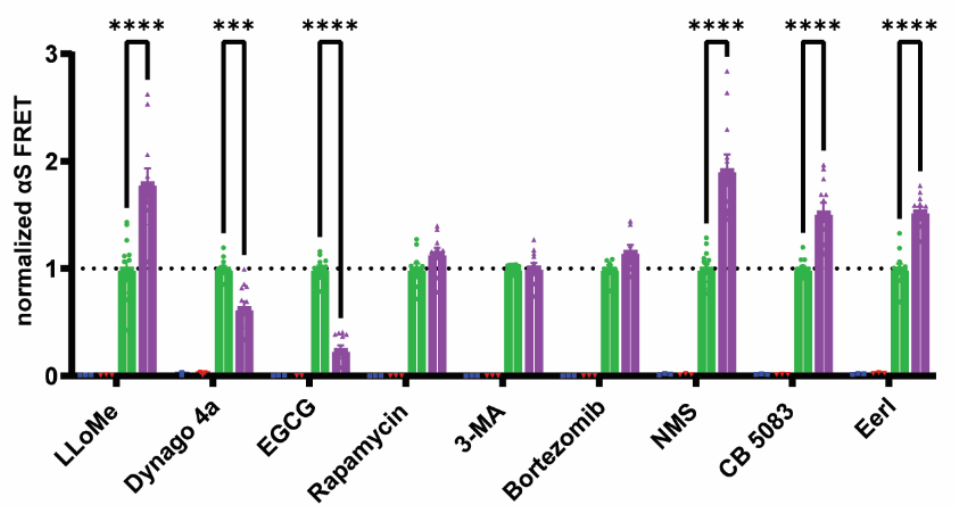

C
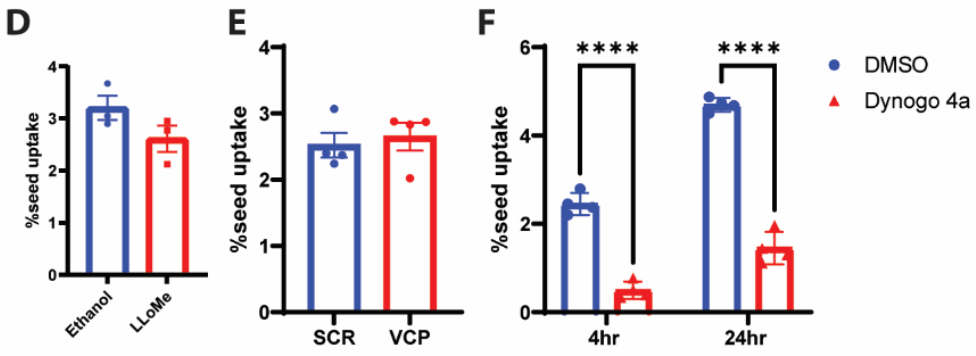

G

H

I
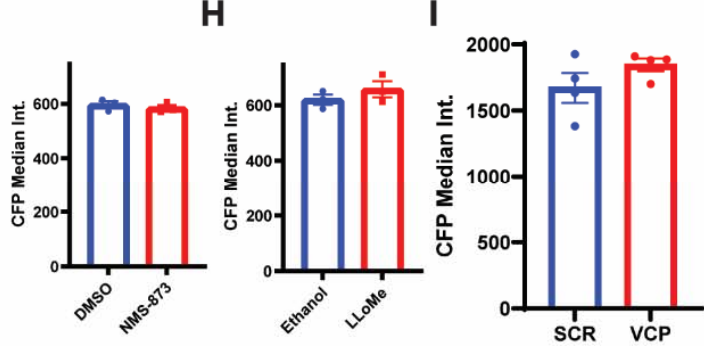

K

Scr VCP siRNA day 2 day 3

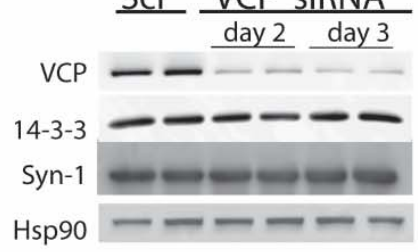

L

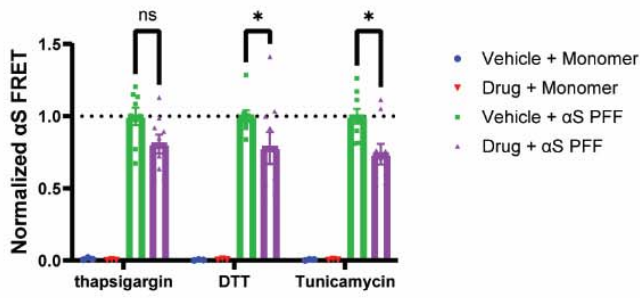

J

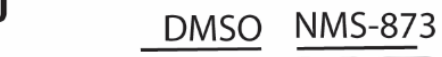

a-tubulin-

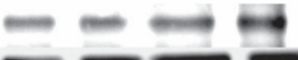

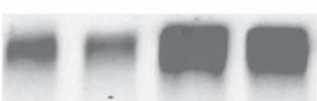

FK-2

GAPDH 
Figuriokiv preprint doi: https://doi.org/10.1101/2021.07.12.452081; this version posted July 13, 2021. The copyright holder for this preprint (which
was not certified by peer review) is the author/funder. All rights reserved. No reuse allowed without permission.

A

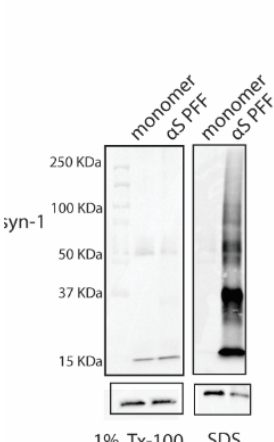

B

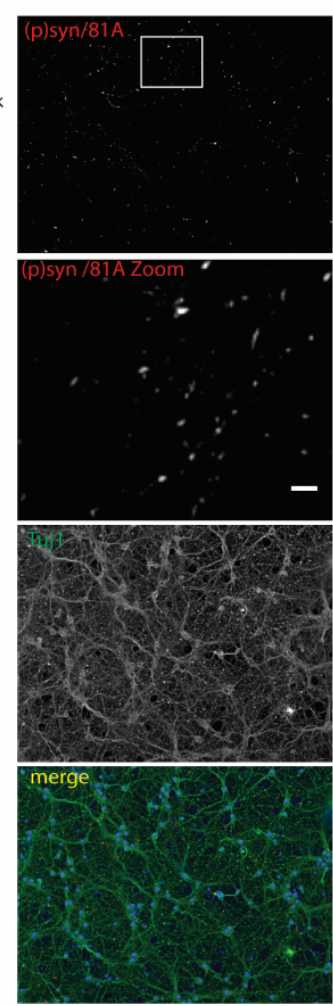

$\mathrm{EtOH}$

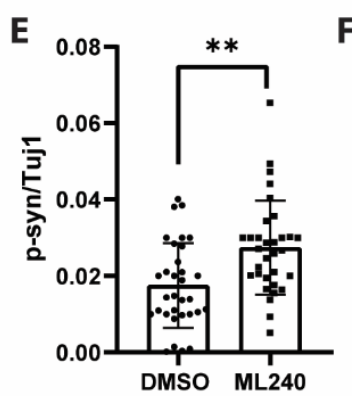

F

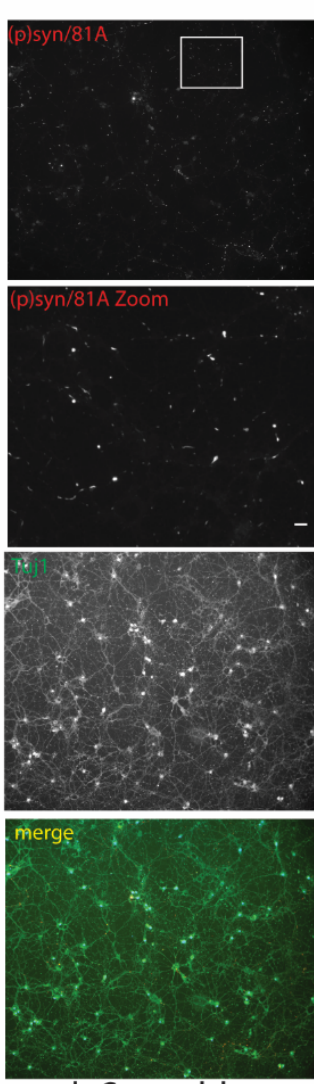

sh Scramble

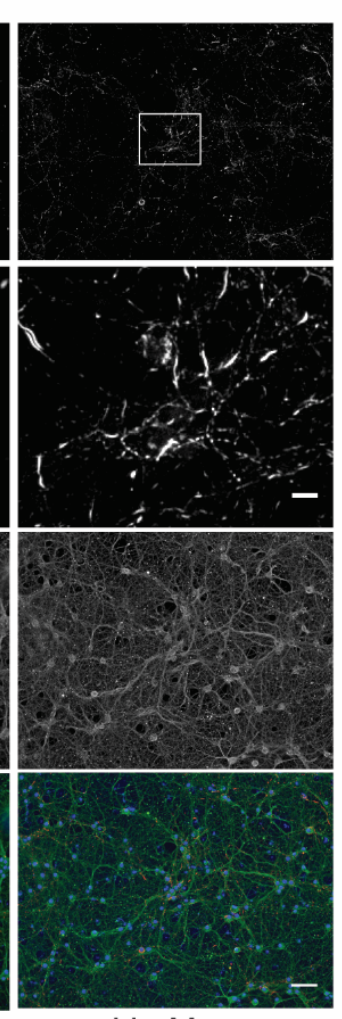

LLoMe
C

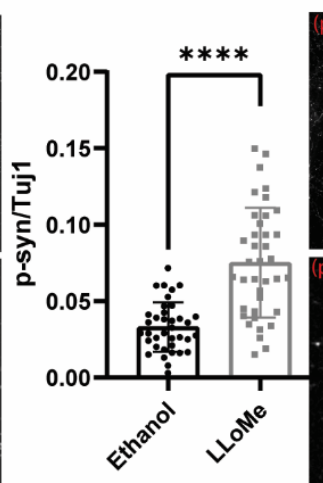

D
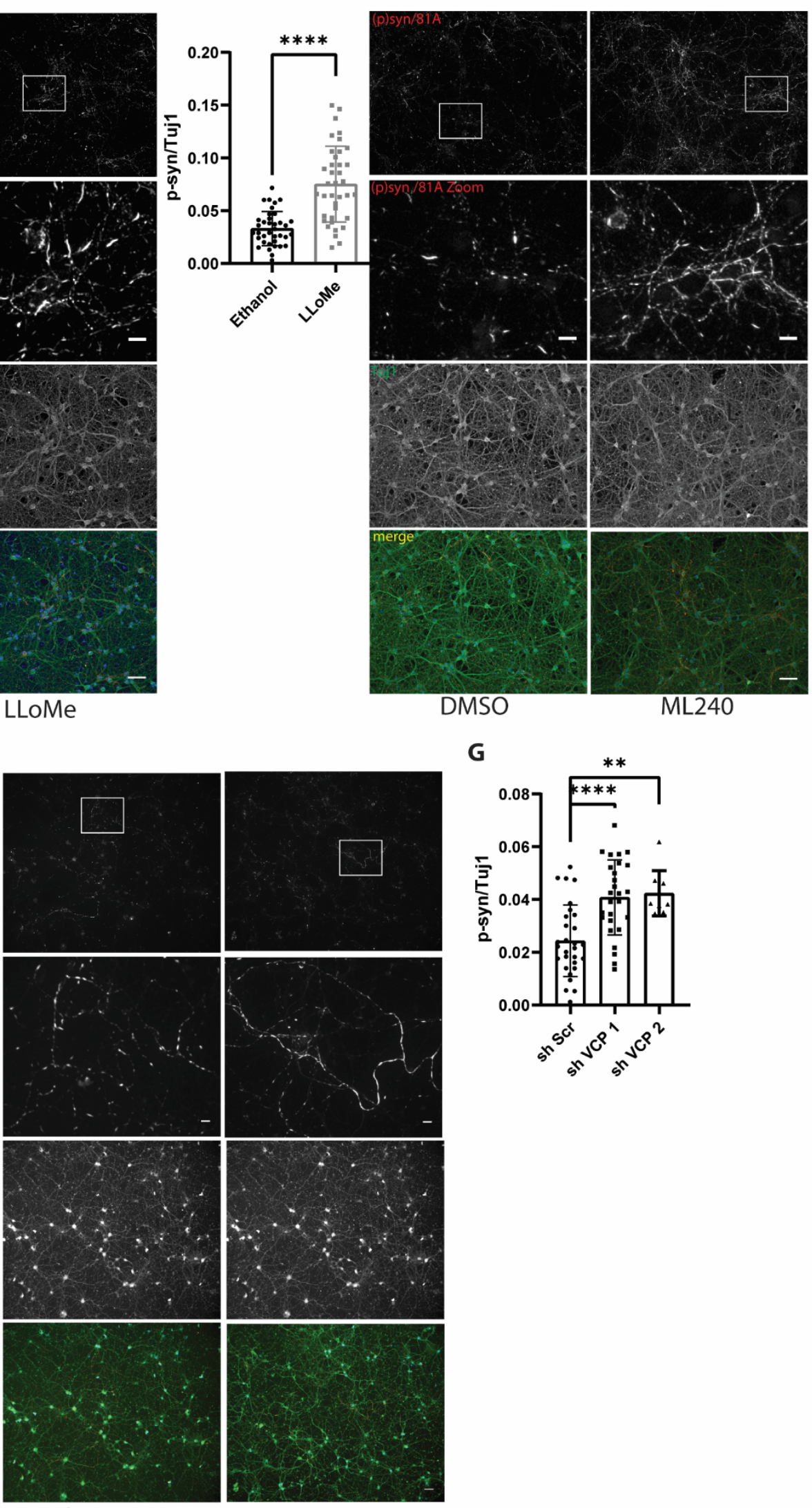

sh VCP 1
G

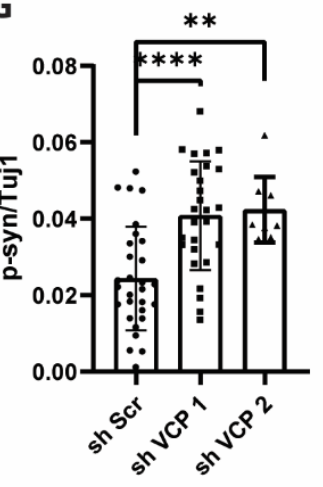

\section{sh VCP 2}


Figure biokiv $q_{\text {eprint doi: https://doi.org/10.1101/2021.07.12.452081; this version posted July 13, 2021. The copyright holder for this preprint (which }}$ was not certified by peer review) is the author/funder. All rights reserved. No reuse allowed without permission.

A

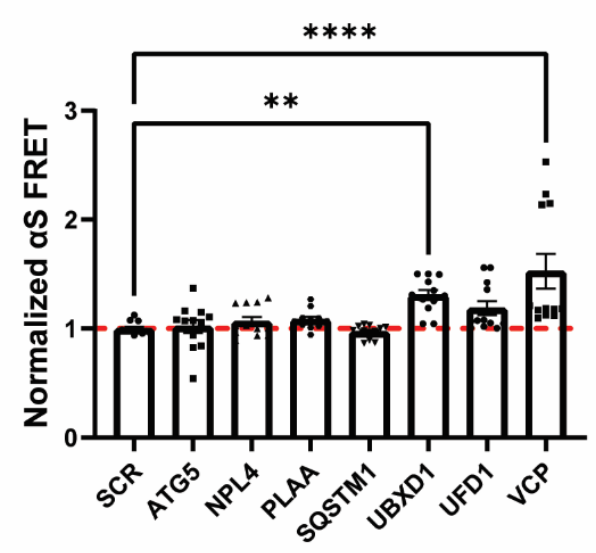

Gene knockdown

B
D

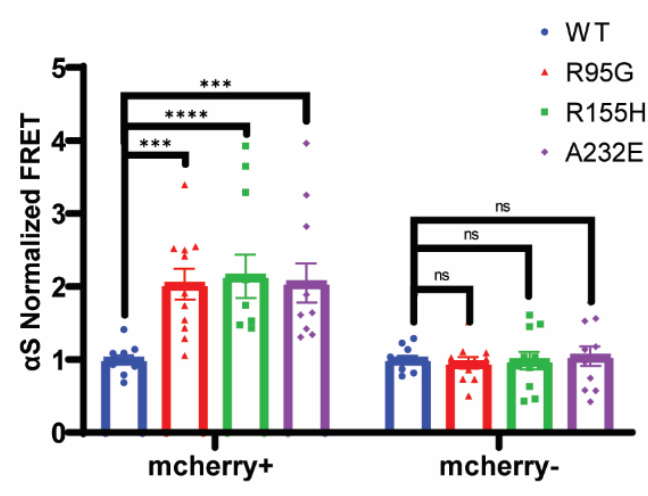

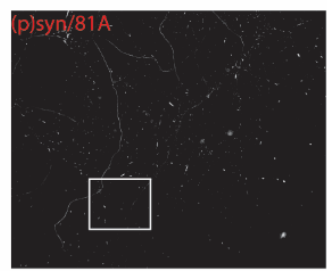
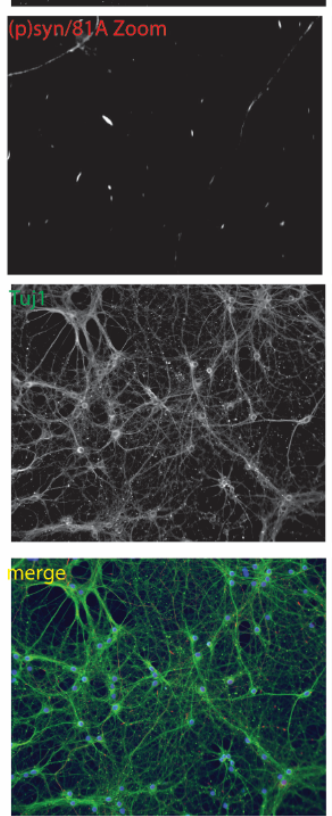

sh Scramble

E
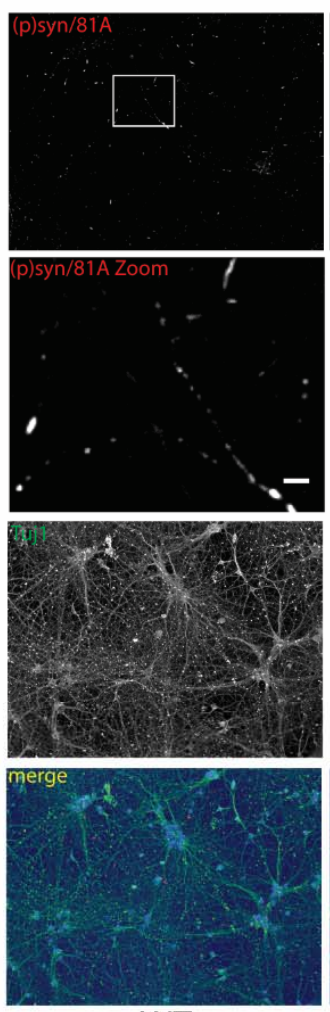

WT
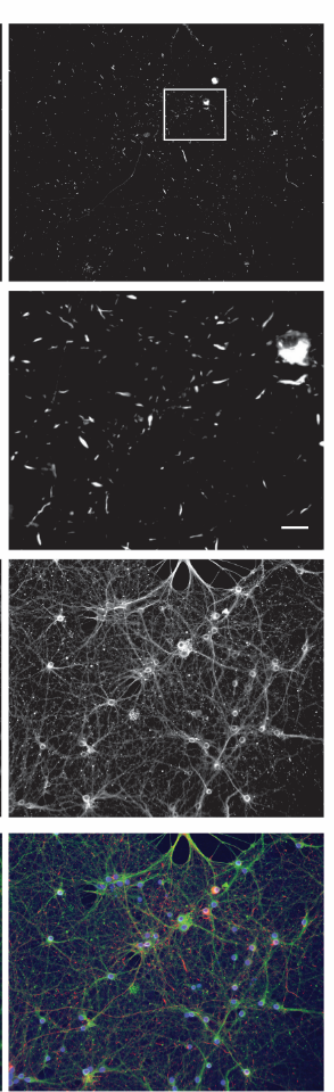

sh UBXD1

F
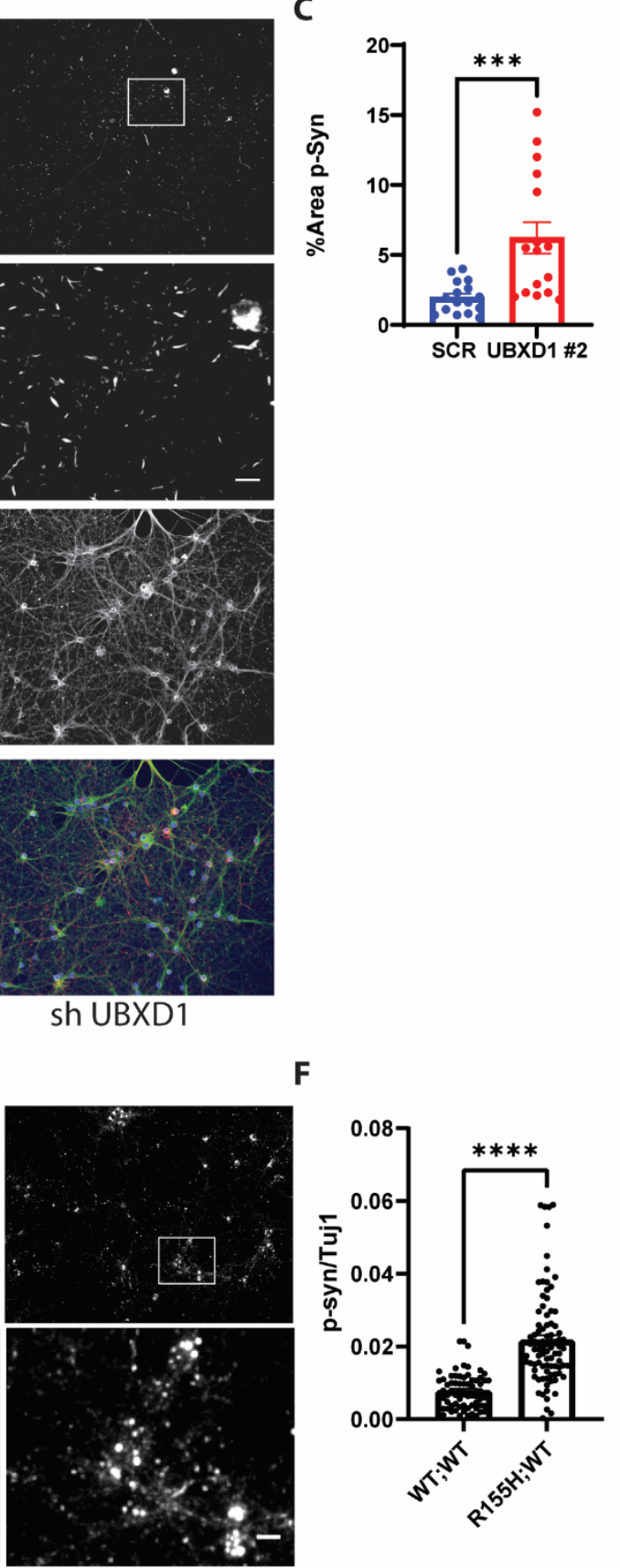
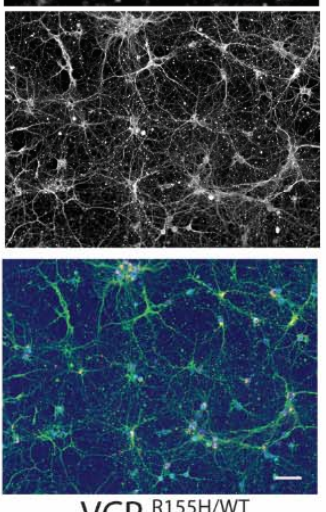

VCP ${ }^{\mathrm{R} 155 \mathrm{H} / \mathrm{WT}}$ 
FigbioRxiv foprint doi: https://doi.org/10.1101/2021.07.12.452081; this version posted July 13, 2021. The copyright holder for this preprint (which Figure 5 was not certified by peer review) is the author/funder. All rights reserved. No reuse allowed without permission.

A C57 CVCP-R155C VCP-R155H

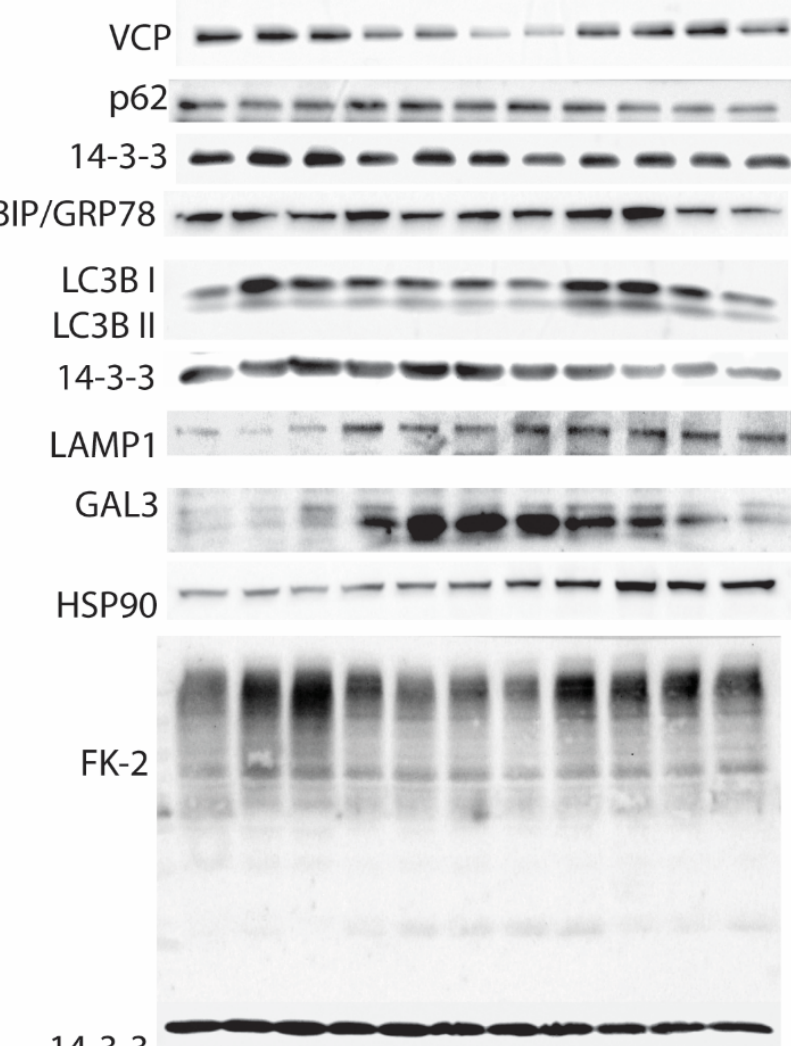

$14-3-3$

B

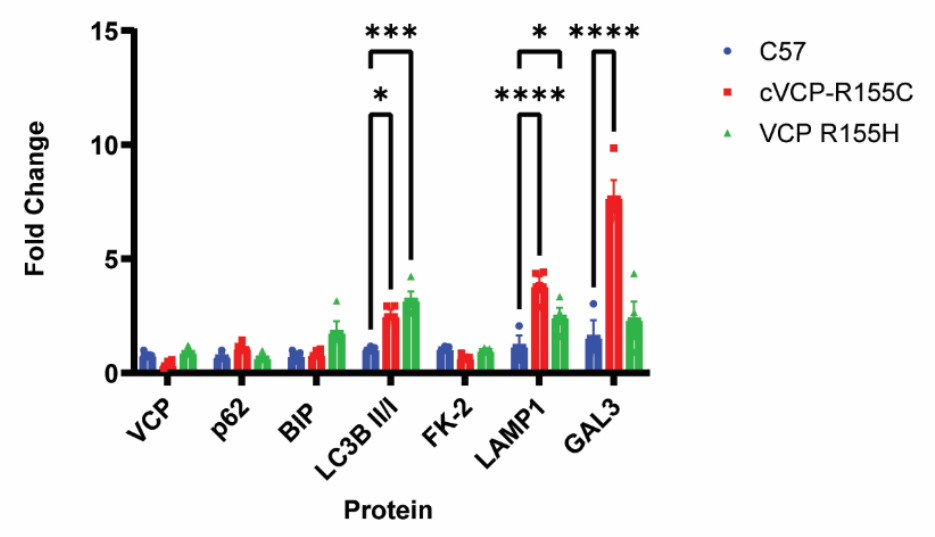

C

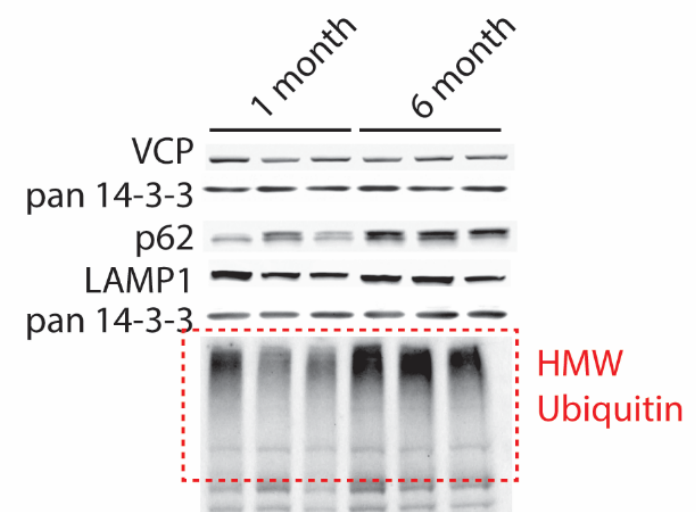

FK-2

pan 14-3-3 - - - - -

Gal-3 - - - - -

pan 14-3-3

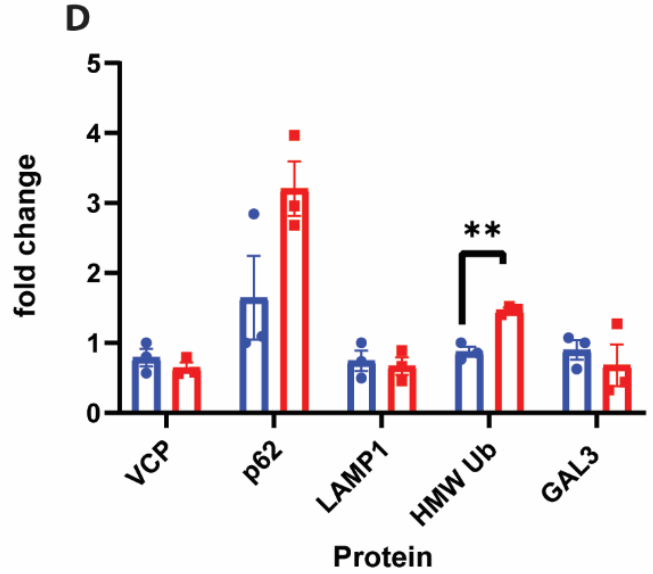


Figure biokxiv print doi: https://doi.org/10.1101/2021.07.12.452081; this version posted July 13,2021 . The copyright holder for this preprint (which
was not certified by peer review) is the author/funder. All rights reserved. No reuse allowed without permission.

A

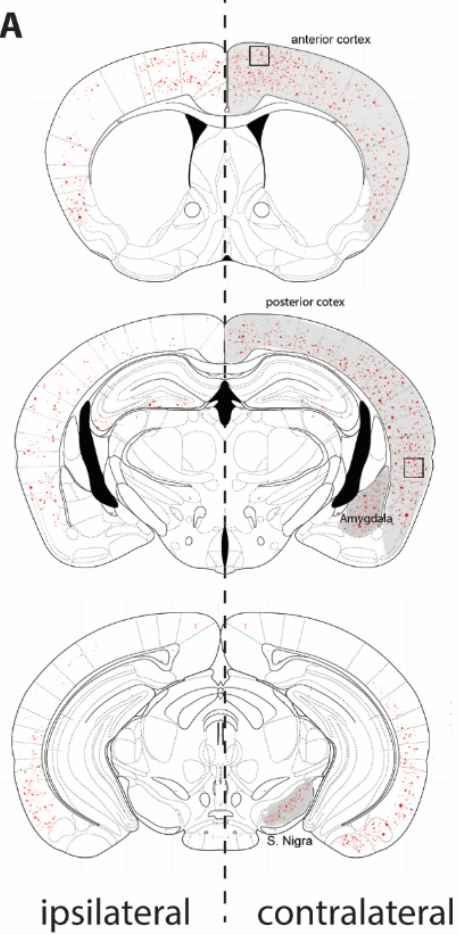

D
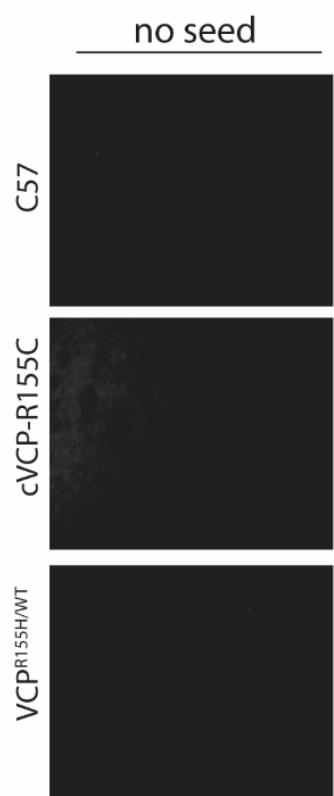

F

Amygdala

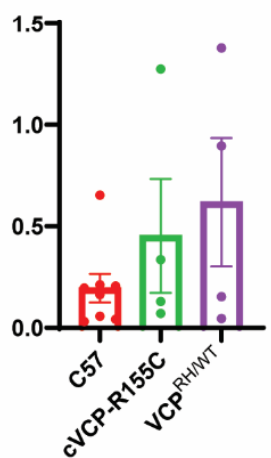

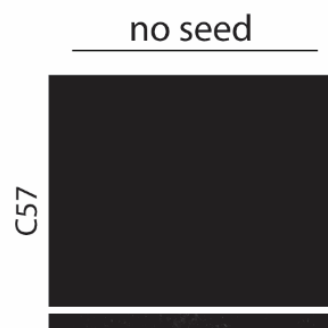
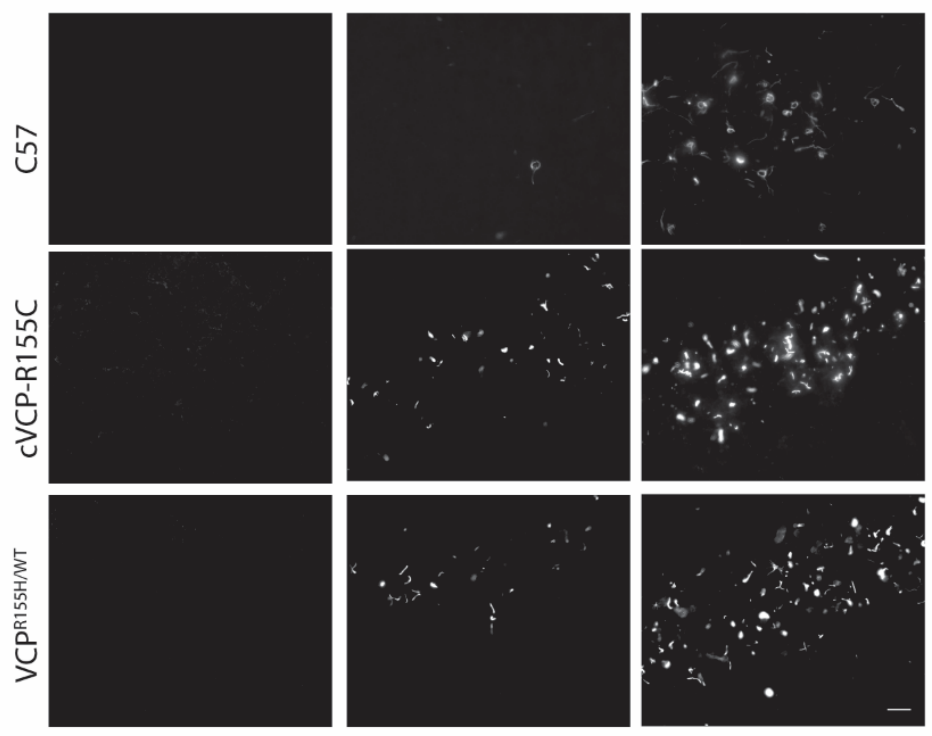

C

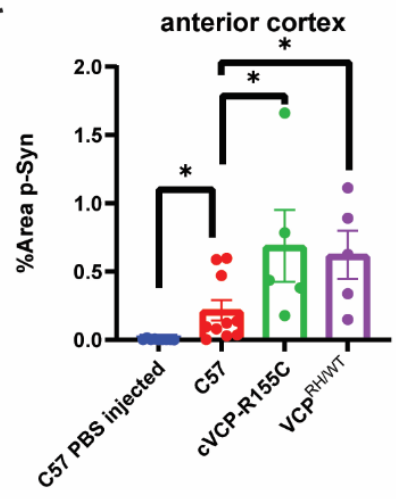

E

with seed
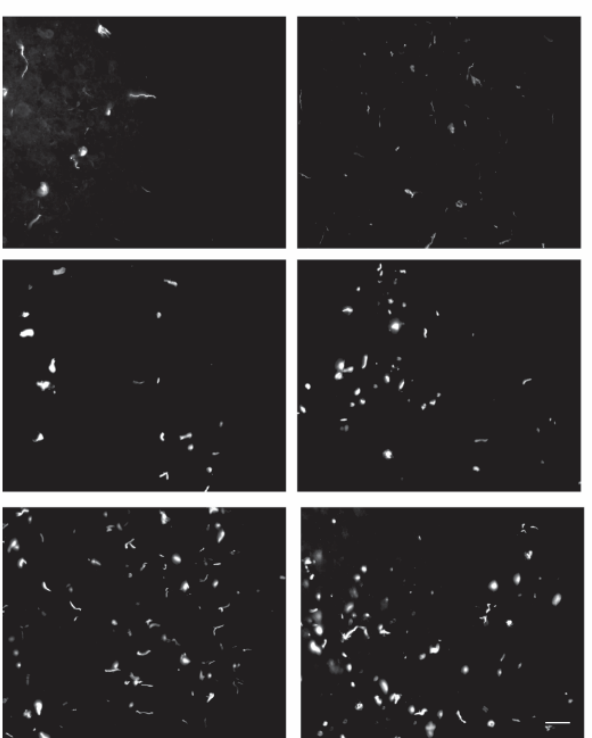

G
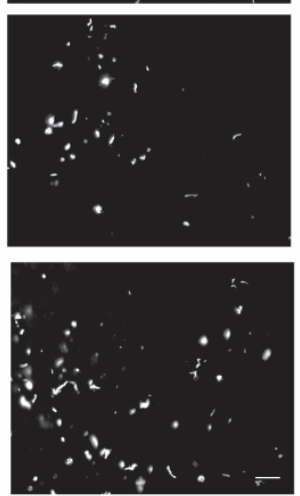

S. Nigra
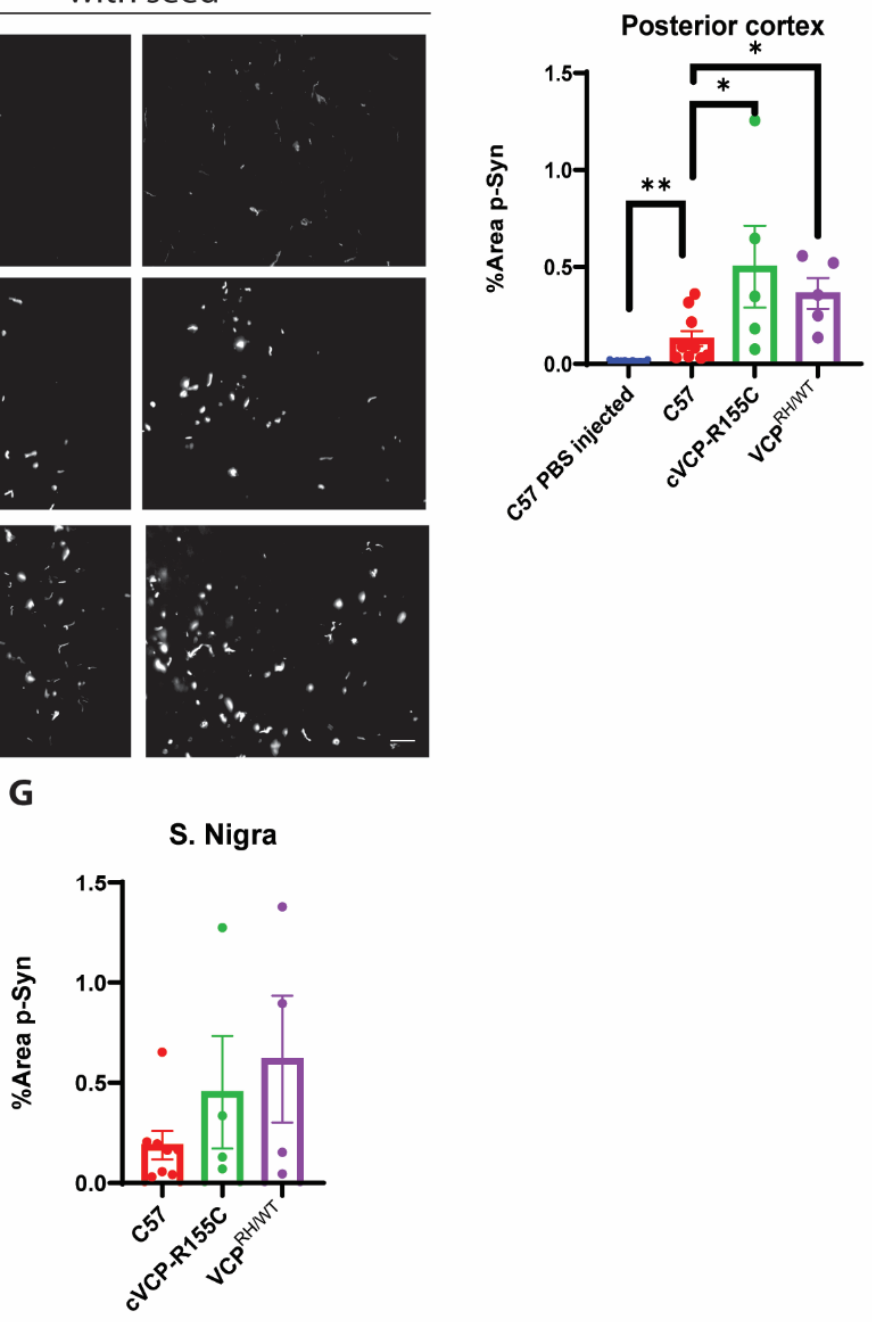


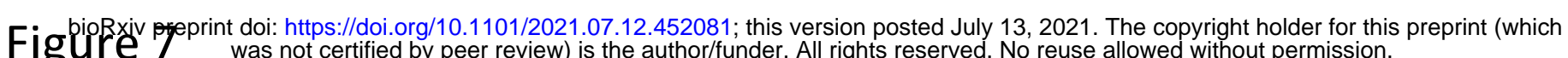
Figure was not certified by peer review) is the author/funder. All rights reserved. No reuse allowed without permission.

A

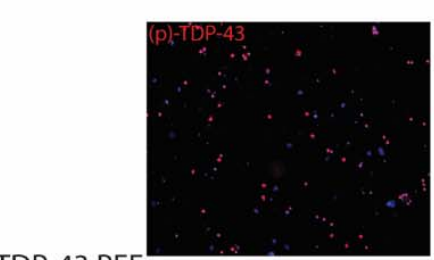

TDP-43 PFF

OnM

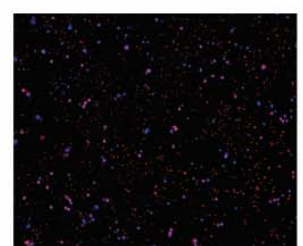

$10 \mathrm{nM}$

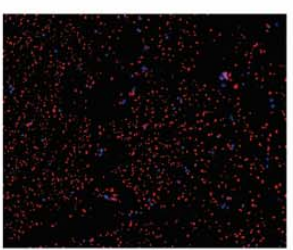

$50 \mathrm{nM}$

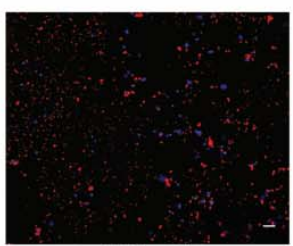

B

C

D
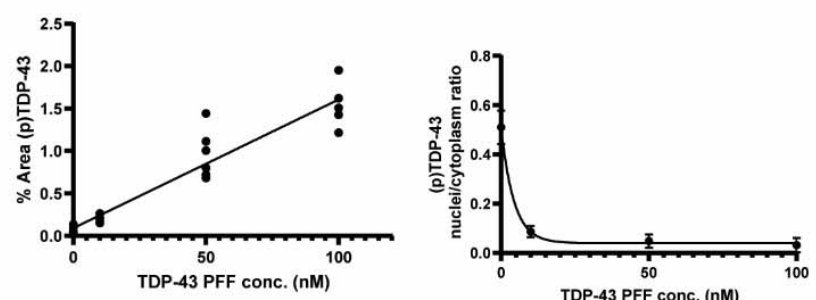

harvest after

1 day -

5 days -

$100 \mathrm{nM}$
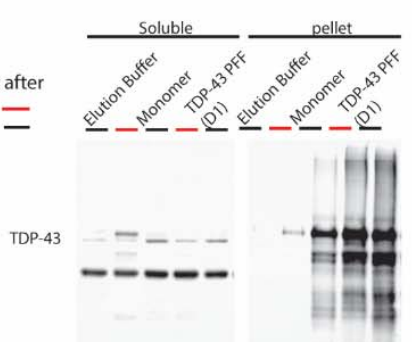

pan 14-3-3- - -

E
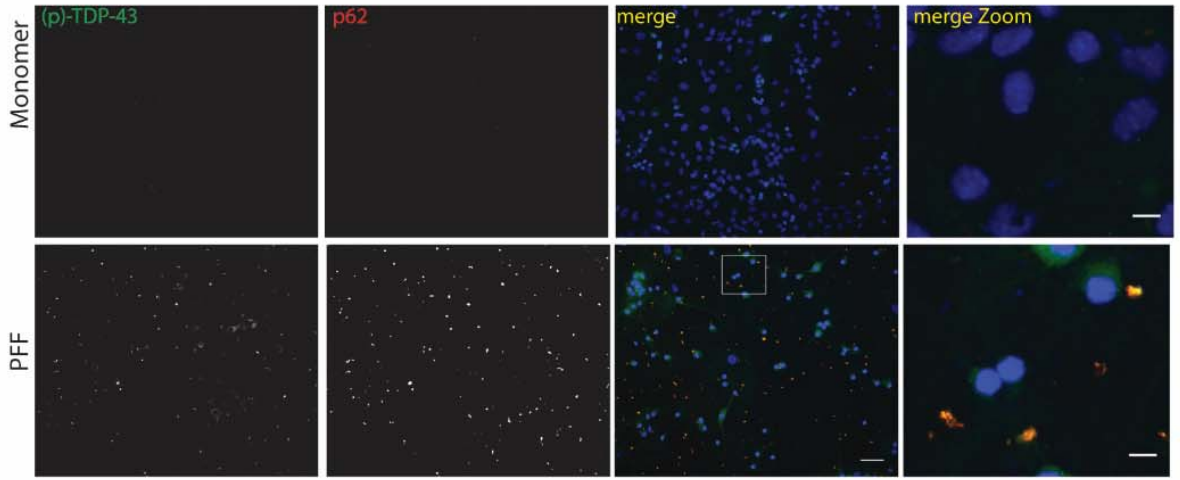

हे
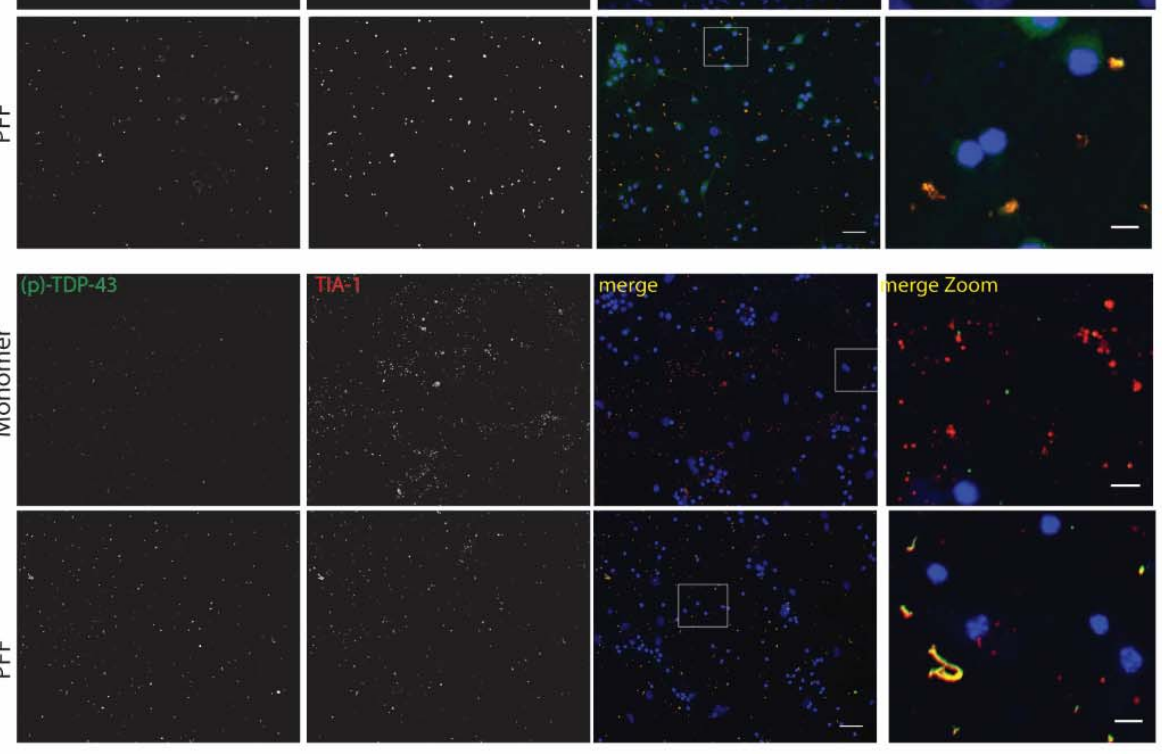

$\mathbf{G}$
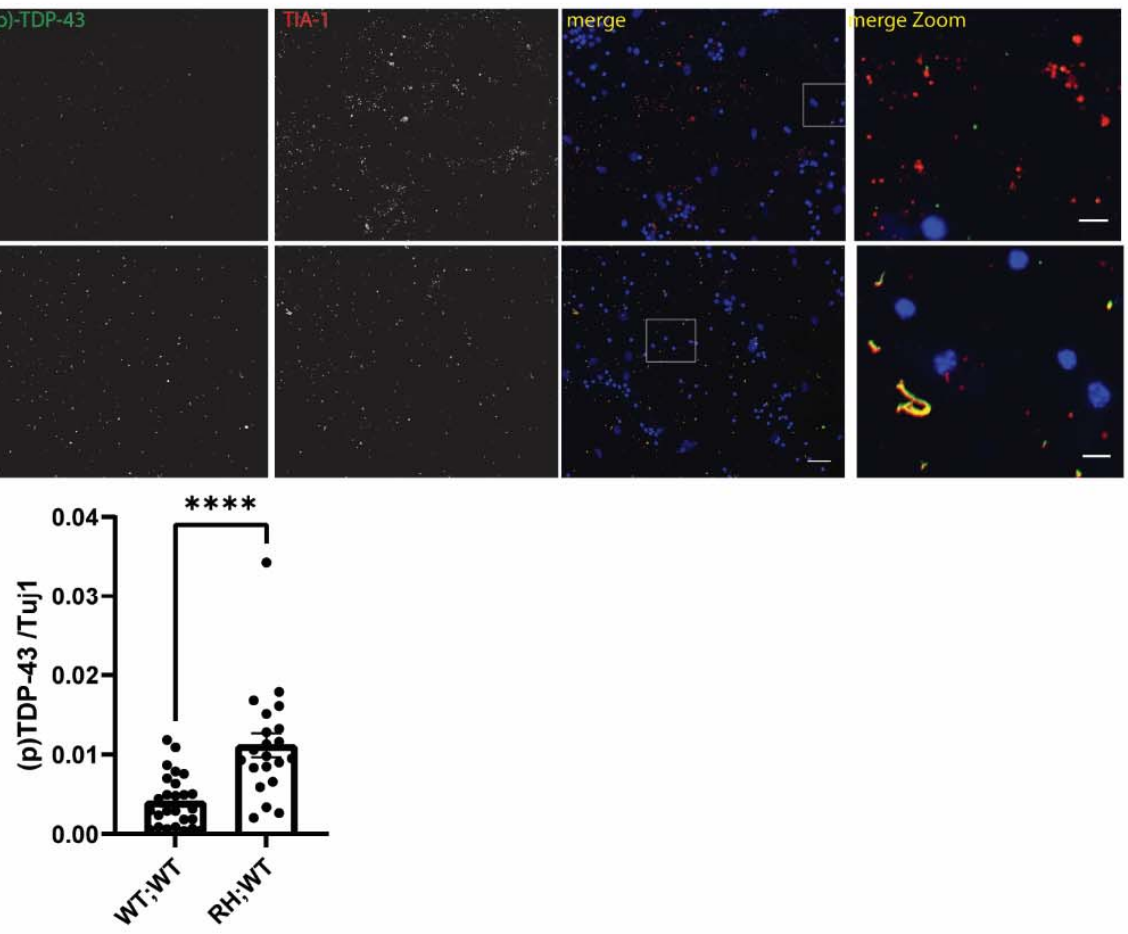

F
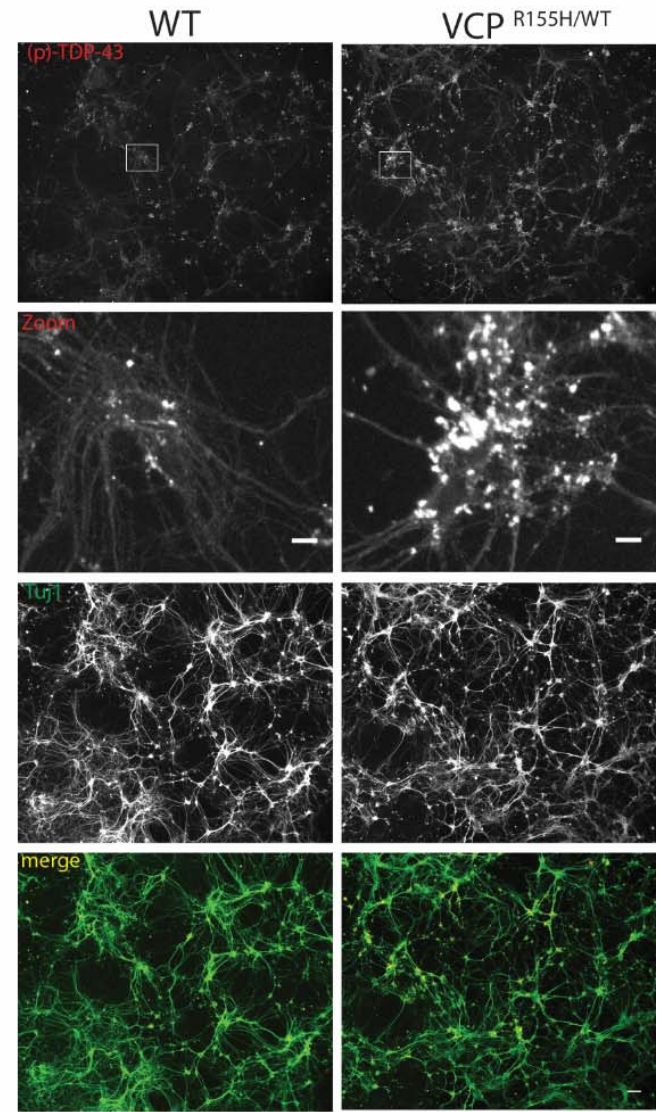